\title{
Dark matter from a vector field in the fundamental representation of $S U(2)_{L}$
}

\author{
Bastian Díaz Sáez, ${ }^{1}$ Felipe Rojas-Abatte, ${ }^{2}$ and Alfonso R. Zerwekh ${ }^{1}$ \\ ${ }^{1}$ Departamento de Física and Centro Científico-Tecnológico de Valparaíso, Universidad Técnica \\ Federico Santa María, Casilla 110-V, Valparaíso, Chile \\ ${ }^{2}$ University of Southampton, Southampton, United Kingdom
}

(Received 30 October 2018; published 24 April 2019)

\begin{abstract}
We explore an extension to the Standard Model which incorporates a vector field in the fundamental representation of $S U(2)_{L}$ as the only nonstandard degree of freedom. This kind of field may appear in different scenarios such as compositeness, gauge-Higgs unification, and extradimensional scenarios. We study the model in which a $Z_{2}$ symmetry is manifiest, making the neutral $C P$-even component of the new vector field a vectorial dark matter candidate. We constraint the parameter space through LEP and LHC data, as well as from current dark matter searches. Additionally, comments on the implications of perturbative unitarity are presented. We find that the model is highly constrained but a small region of the parameter space can provide a viable DM candidate. On the other hand, unitarity demands an UV completion at an scale below $10 \mathrm{TeV}$. Finally we contrast our predictions on mono-jet, - $Z$, -Higgs production with the ones obtained in the inert two Higgs doublet model.
\end{abstract}

DOI: 10.1103/PhysRevD.99.075026

\section{INTRODUCTION}

It is generally acknowledged that the Standard Model (SM) is incomplete, despite its impressive and unexpected phenomenological success. Its lack of a dark matter (DM) candidate and the impossibility of naturally generating within its framework tiny but nonvanishing neutrino masses are usually among the reasons invoked to illustrate such an incompleteness. Additionally, and maybe more dramatically, even the dynamical origin of the electroweak scale is not completely understood in within the SM. This has motivated, over the years, the construction of many extensions of SM. However, the very precise measurement made at LEP during the 1990s already taught us that the new physics has to be subtle, making the construction of consistent and complete new physics models, a formidable task. Of course, the lack of evidence of any kind of nonstandard phenomena at the LHC has put stronger constraints and has made the labor of model-builders even more difficult.

Under these circumstances, it seems wise to take a less ambitious approach. In the search of some dark matter signal, both effective field theories (EFT) (see, e.g., [1-8]) and simplified models frameworks (see, e.g., [9-13]) have been used as a guide of search. In the latter framework, both

Published by the American Physical Society under the terms of the Creative Commons Attribution 4.0 International license. Further distribution of this work must maintain attribution to the author(s) and the published article's title, journal citation, and DOI. Funded by SCOAP . scalar and fermion dark matter has been the most explored line by their simplicity (for a classification under SM quantum numbers see [14]). However, it has been shown that vector bosons may perfectly play the role of dark matter, most of them motivated from hidden gauge sectors [15-24], extra large dimensions [25], little Higgs model [26] and from a linear sigma model [27]. Recently, the neutral component of an electroweak vector multiplet has been shown to be a good dark matter candidate, such as multiplets transforming in the adjoint representation [28], and in the fundamental one in the context of 331 models $[29,30]$ and in Gauge-Higgs unification framework [31].

In this work, taking an agnostic UV-completion approach, we consider a simplified model which takes an electroweak vector multiplet transforming in the fundamental representation of $S U(2)_{L}$ with hypercharge $1 / 2$, leading naturally a dark matter candidate due to an accidental $Z_{2}$ symmetry. We introduce what we call the dark vector doublet model (DVDM), and we contrast this possibility with theoretical and experimental constrains. Interestingly, the new fields couple to the SM bosons $(Z$, $W^{ \pm}$, photon and the Higgs boson) but, as we will see, not to the SM fermions if we only consider up to renormalizable operators. We constaint the model through experimental data such as LEP, LHC and dark matter probes. We show that our vector dark matter field can account for the total observed dark matter abundance for masses above $840 \mathrm{GeV}$, approximately.

Finally, DM cross section and missing transverse energy at the LHC are observables capables to discriminate among different signals. In view of the similarities between our 
model and the well known inert two Higgs doublet model (i2HDM) [32-37], we compare the cross section and missing energy distribution shapes for monojet, $-Z$ and Higgs signals in both models.

The paper is organized in the following way. In Sec. II we describe our model and we specify under what conditions a dark matter candidate appears. The space parameter is discussed in Sec. III. We present as well all the relevant theoretical and experimental constraints we used to study the model in Sec. IV. Next, in Sec. V a deep description of the DM phenomenology of the model with a full scan of the parameter space is shown accompanied with a brief discussion of DM searches at LHC. As a complementary analysis, in Sec. VI we discuss perturbative unitarity. Finally we present our conclusions in Sec. VII. In the Appendix we show more details about the discussion of perturbative unitarity.

\section{THE LAGRANGIAN}

As we announced, we extend the SM by introducing a new set of vector fields in a single-representation of the standard gauge group $S U(3)_{c} \times S U(2)_{L} \times U(1)_{Y}$ :

$$
V_{\mu}=\left(\begin{array}{c}
V_{\mu}^{+} \\
V_{\mu}^{0}
\end{array}\right)=\left(\begin{array}{c}
V_{\mu}^{+} \\
\frac{V_{\mu}^{1}+i V_{\mu}^{2}}{\sqrt{2}}
\end{array}\right),
$$

transforming as $(\mathbf{1}, \mathbf{2}, 1 / 2)$. Notice that we are assuming that $V_{\mu}$ has the same weak-isospin and hypercharge as the Higgs doublet $(\phi)$. In other words, we are assuming that $V_{\mu}^{1}$ and $V_{\mu}^{2}$ are neutral states. The most general Lagrangian respecting the SM gauge symmetry containing this new vectors with operators up to dimension four is

$$
\begin{aligned}
\mathcal{L} & =-\frac{1}{2}\left(D_{\mu} V_{\nu}-D_{\nu} V_{\mu}\right)^{\dagger}\left(D^{\mu} V^{\nu}-D^{\nu} V^{\mu}\right)+M_{V}^{2} V_{\mu}^{\dagger} V^{\mu} \\
& +\lambda_{2}\left(\phi^{\dagger} \phi\right)\left(V_{\mu}^{\dagger} V^{\mu}\right)+\lambda_{3}\left(\phi^{\dagger} V_{\mu}\right)\left(V^{\mu \dagger} \phi\right)+\lambda_{4}\left(\phi^{\dagger} V_{\mu}\right)\left(\phi^{\dagger} V^{\mu}\right) \\
& +\alpha_{1} \phi^{\dagger} D_{\mu} V^{\mu}+\alpha_{2}\left(V_{\mu}^{\dagger} V^{\mu}\right)\left(V_{\nu}^{\dagger} V^{\nu}\right)+\alpha_{3}\left(V_{\mu}^{\dagger} V^{\nu}\right)\left(V_{\nu}^{\dagger} V^{\mu}\right) \\
& +i g \kappa_{1} V_{\mu}^{\dagger} W^{\mu \nu} V_{\nu}+i \frac{g^{\prime}}{2} \kappa_{2} V_{\mu}^{\dagger} B^{\mu \nu} V_{\nu}+\text { H.c. }
\end{aligned}
$$

where $B^{\mu \nu}$ is the Abelian $U(1)_{Y}$ field strength, and $W^{\mu \nu}=$ $W^{\mu \nu a} \frac{\tau^{a}}{2}$ is the non-Abelian $S U(2)_{L}$ field strength. In principle, all the free parameters, $\lambda_{i}, \alpha_{i}$ for $i=1,2,3$ may be complex. The parameters $\kappa_{1}$ and $\kappa_{2}$ are analogous to the well-known anomalous couplings in the context of vector leptoquark models.

Interestingly, it is not possible to couple the new vector boson to the standard fermions with renormalizable operators. For example, without loss of generality, let us suppose a Lorentz invariant Yukawa-like coupling between
SM first generation of leptons and the vector doublet. Then, consider the following vector and axial vector couplings,

$$
\mathcal{L} \supset \bar{L} \gamma^{\mu}\left(g^{V}-g^{A} \gamma^{5}\right) e_{R} V_{\mu},
$$

where $g^{V}$ and $g^{A}$ are arbitrary coupling constants. Considering the chirality projectors $P_{L, R}$, the Lagrangian (3) may be rewritten as

$$
\begin{aligned}
\mathcal{L} & =\left(\bar{\nu}_{e} \bar{e},\right) P_{R} \gamma^{\mu}\left(g^{V}-g^{A} \gamma^{5}\right) P_{R} e V_{\mu}, \\
& =\left(\bar{\nu}_{e} \bar{e}\right) \gamma^{\mu}\left(g^{V}-g^{A} \gamma^{5}\right) P_{L} P_{R} e V_{\mu} \\
& =0
\end{aligned}
$$

where we have used $\left\{\gamma^{\mu}, \gamma^{5}\right\}=0$ and $P_{L} P_{R}=0$.

On the other hand, the term proportional to $\alpha_{1}$ is allowed by the symmetry, and it is an operator of dimension three and the only one linear in $V_{\mu}$. In principle, this term would introduce a mixing between the SM gauge bosons and the new vector states. However, it is possible to set up it to zero naturally because an accidental $Z_{2}$ symmetry arises in the model. Due to this discrete symmetry, the new heavy vectors do not mix with the electroweak gauge bosons so there are no corrections at tree level to $M_{W}$ and $M_{Z}$, keeping untouch the $\rho$-parameter at tree level. Everything goes like in the SM for the electroweak gauge bosons. Therefore $\Delta S=\Delta T=0$ at tree level in our model, and any correction will appear at loop level.

Finally, the terms in the last line of (2) are allowed by the symmetry. However the values of their coupling constants $\left(\kappa_{1}\right.$ and $\left.\kappa_{2}\right)$ are not fixed by the symmetries. In this paper, we work in the simplified case where $\kappa_{1}=\kappa_{2}=1$. This choice is consistent with the hypercharge assigned to $V_{\mu}$ and agrees with what happen in vector leptoquarks models, where the ultraviolet gauge completion and unitarity arguments fixes the values of those parameters to one [38]. In other words, if we allow for values different to one, there appear the coupling among the photon $A_{\mu}$ and the two neutral vector $V^{1}$ and $V^{2}$, implying the latter fields now get an electric charge.

\section{DARK MATTER CANDIDATE}

As we explained above, in the limit when $\alpha_{1}$ vanish, the model acquires an additional $Z_{2}$ discrete symmetry allowing the stability of the lightest odd particle (LOP). If the LOP happens to be a neutral component of $V_{\mu}$ (as it must be for cosmological reasons) then it constitutes a good DM candidate. In this case, the Lagrangian (2) reduces to: 


$$
\begin{aligned}
\mathcal{L}= & -\frac{1}{2}\left(D_{\mu} V_{\nu}-D_{\nu} V_{\mu}\right)^{\dagger}\left(D^{\mu} V^{\nu}-D^{\nu} V^{\mu}\right)+M_{V}^{2} V_{\mu}^{\dagger} V^{\mu} \\
& -\alpha_{2}\left(V_{\mu}^{\dagger} V^{\mu}\right)\left(V_{\nu}^{\dagger} V^{\nu}\right)-\alpha_{3}\left(V_{\mu}^{\dagger} V^{\nu}\right)\left(V_{\nu}^{\dagger} V^{\mu}\right) \\
& -\lambda_{2}\left(\phi^{\dagger} \phi\right)\left(V_{\mu}^{\dagger} V^{\mu}\right)-\lambda_{3}\left(\phi^{\dagger} V_{\mu}\right)\left(V^{\mu^{\dagger}} \phi\right) \\
& -\frac{\lambda_{4}}{2}\left[\left(\phi^{\dagger} V_{\mu}\right)\left(\phi^{\dagger} V^{\mu}\right)+\left(V^{\mu^{\dagger}} \phi\right)\left(V_{\mu}^{\dagger} \phi\right)\right] \\
& +i \frac{g^{\prime}}{2} V_{\mu}^{\dagger} B^{\mu \nu} V_{\nu}+i g V_{\mu}^{\dagger} W^{\mu \nu} V_{\nu} .
\end{aligned}
$$

Curiously, this Lagrangian is rather similar to the i2HDM $[33,37,39]$ where the extra scalar doublet is replaced by the new vector doublet.

The Lagrangian 4 contain six free parameters ${ }^{1}$ which we labeled as $\lambda_{2}, \lambda_{3}, \lambda_{4}$ for quartic coupling involving interactions between SM-Higgs field and the new vector field, a mass term $M_{V}$, and $\alpha_{2}, \alpha_{3}$ for quartic couplings of pure interactions among the vector fields. These latter selfinteracting terms are not relevant for the experimental constraints and dark matter phenomenology done in this paper, therefore from now on we will not consider them, However, self-interacting particle dark matter can be relevant in related fields such as astrophysical structures [40].

After the electroweak symmetry breaking, the tree level mass spectrum of the new sector is

$$
\begin{gathered}
M_{V^{ \pm}}^{2}=\frac{1}{2}\left[2 M_{V}^{2}-v^{2} \lambda_{2}\right], \\
M_{V^{1}}^{2}=\frac{1}{2}\left[2 M_{V}^{2}-v^{2}\left(\lambda_{2}+\lambda_{3}+\lambda_{4}\right)\right], \\
M_{V^{2}}^{2}=\frac{1}{2}\left[2 M_{V}^{2}-v^{2}\left(\lambda_{2}+\lambda_{3}-\lambda_{4}\right)\right] .
\end{gathered}
$$

The term proportional to $\lambda_{4}$ makes the splitting between the physical masses of the two neutral states. For phenomenological proposes we will work in a different base of free parameters

$$
M_{V^{1}}, \quad M_{V^{2}}, \quad M_{V^{ \pm}}, \quad \lambda_{L}
$$

where $\lambda_{L}=\lambda_{2}+\lambda_{3}+\lambda_{4}$ is, as we will see, the effective coupling controlling the interaction between the SM Higgs and $V^{1}$. It is convenient to write the quartic coupling and the mass parameter as a function of the new free parameters

\footnotetext{
${ }^{1}$ We assume that all the free parameters are real, otherwise, the new vector sector may introduce $C P$-violation sources. In this work we do not deal with that interesting possibility.
}

$$
\begin{aligned}
& \lambda_{2}=\lambda_{L}+2 \frac{\left(M_{V^{1}}^{2}-M_{V^{ \pm}}^{2}\right)}{v^{2}}, \quad \lambda_{3}=\frac{2 M_{V^{ \pm}}^{2}-M_{V^{1}}^{2}-M_{V^{2}}^{2}}{v^{2}}, \\
& \lambda_{4}=\frac{M_{V^{2}}^{2}-M_{V^{1}}^{2}}{v^{2}}, \quad M_{V}^{2}=M_{V^{1}}^{2}+\frac{v^{2} \lambda_{L}}{2} .
\end{aligned}
$$

For future convenience, it will be useful to introduce

$$
\lambda_{R} \equiv \lambda_{2}+\lambda_{3}-\lambda_{4}=\lambda_{L}+\frac{2\left(M_{V^{2}}^{2}-M_{V^{1}}^{2}\right)}{v^{2}},
$$

which is not a new free parameter, but it is the effective coupling constant which governs the $H V^{2} V^{2}$ interaction.

It is important to mention that because the new vector field have the same quantum numbers as the SM-Higgs field, the two neutral vectors have opposite $C P$-parities. However we can switch their parity just making a change of bases $V_{\mu} \rightarrow i V_{\mu}$ and then relabel each field as $V_{\mu}^{1} \rightarrow V_{\mu}^{2}$ and $V_{\mu}^{2} \rightarrow V_{\mu}^{1}$ and still obtain the same phenomenology. Therefore, without loss of generality, we will choose $V_{\mu}^{1}$ as the LOP turning it into our dark matter candidate. Following the same line, to make sure that $V_{\mu}^{1}$ is the lightest state of the new sector, we can find some restrictions that the quartic couplings must follow to satisfy this condition. Considering this we can stress that

$$
\begin{aligned}
& M_{V^{2}}^{2}-M_{V^{1}}^{2}>0 \Rightarrow \lambda_{4}>0, \\
& M_{V^{ \pm}}^{2}-M_{V^{1}}^{2}>0 \Rightarrow \lambda_{3}+\lambda_{4}>0 .
\end{aligned}
$$

In order to have a weakly interacting model, we set that all the couplings parameters must satisfy

$\left|\lambda_{i}\right|<4 \pi \wedge\left|\alpha_{j}\right|<4 \pi \quad(i=2,3,4 ; \quad j=2,3)$.

We implemented this model using the LANHEP [41] package and we used CALCHEP [42] and MICROMEGAs [43-45] for collider and DM phenomenology calculations, respectively.

\section{COLLIDER AND DARK MATTER CONSTRAINTS}

\section{A. LEP limits}

Considering that the coupling between the SM gauge bosons and the dark sector is fixed by gauge invariance, the only way to avoid deviations from precise LEP-I constraints on $W$ and $Z$ widths [46,47] is to demand that the channels $Z \rightarrow V^{1} V^{2}, V^{+} V^{-}$and $W^{ \pm} \rightarrow V^{1} V^{ \pm}, V^{2} V^{ \pm}$are kinematically not open. This leads to the following conditions on the masses

$$
\begin{array}{lr}
M_{V^{1}}+M_{V^{ \pm}}>M_{W^{ \pm}}, & M_{V^{2}}+M_{V^{ \pm}}>M_{W^{ \pm}}, \\
M_{V^{1}}+M_{V^{2}}>M_{Z}, & 2 M_{V^{ \pm}}>M_{Z} .
\end{array}
$$



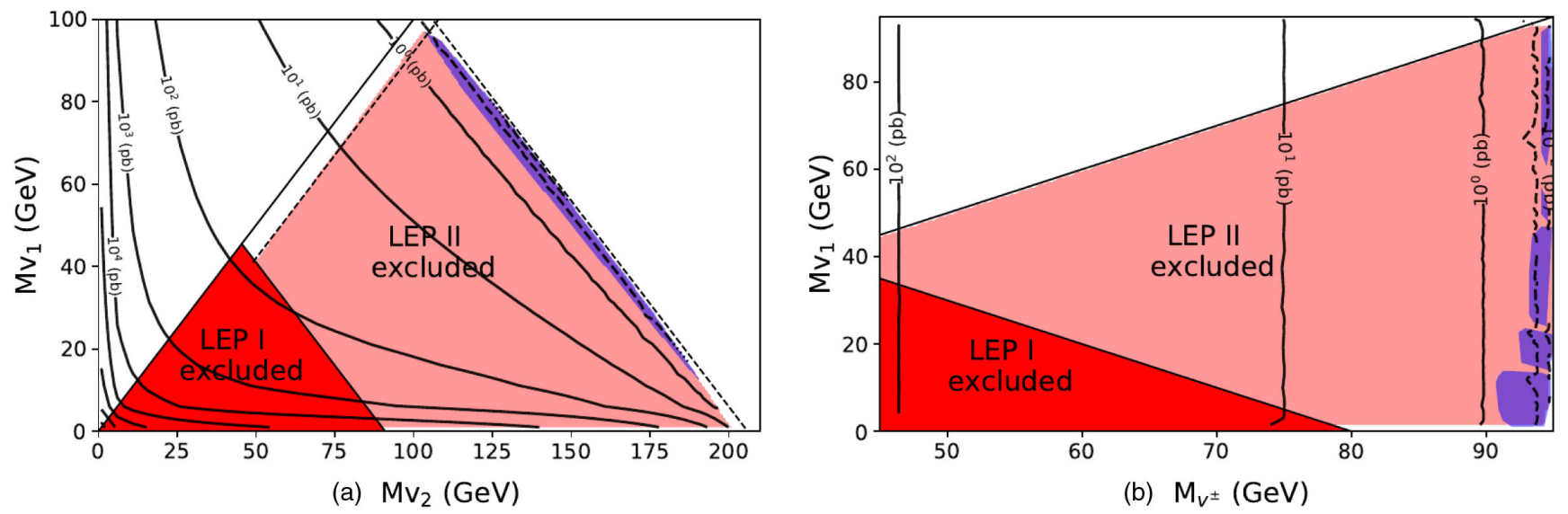

FIG. 1. (a) Allowed mass region for neutral vectors based on 95\% C.L. upper limits on $e^{+} e^{-} \rightarrow \tilde{\chi}_{1}^{0} \tilde{\chi}_{2}^{0}$ cross section at $\sqrt{s}=189$ GeV [50]. The solid black contour lines indicate the production cross section $e^{+} e^{-} \rightarrow V^{1} V^{2}$ at LEP. The red(blue) zones are forbidden (allowed) by LEP-II data. The red shaded region is excluded by LEP-I data on the $Z$ boson width [see (13)]. (b) Allowed mass region for charged and neutral vector based on 95\% C.L. upper-limits on $e^{+} e^{-} \rightarrow \tilde{\chi}_{1}^{+} \tilde{\chi}_{2}^{-}$cross section at $\sqrt{s}=189 \mathrm{GeV}$ [51].

On the other hand, bounds on supersymmetric particles searches at LEP have been very useful to constrain other models beyond the SM. In particular, LEP-II limits on neutralinos and charginos has been used to constraint the inert doublet model (i2HDM) [48,49]. Although there are some differences in the number of Feynman diagrams and the spin involved in the processes, the kinematical efficiencies among the two result to be quite similar, allowing us to recast the experimental bounds.

In view of the identical topologies in the processes of the i2HDM and our model, it seems natural to extend the LEP bounds to our vectorial case. The concern is whether the efficiencies of the vectorial signals are similar to the SUSY ones. In the case of neutral state production, the process $e^{+} e^{-} \rightarrow Z \rightarrow V^{1} V^{2}$ shows a distribution more isotropic and similar to neutralinos, because both cases, having intrinsic spin, have the ability to conserve angular momentum. Scalars, on the other hand, which are produced through the same topology as the vector ones, $e^{+} e^{-} \rightarrow Z \rightarrow H^{0} A^{0}$, are produced in $p$-waves, making the scalars have large transverse momentum. Additionally, as it has been shown in [48], the angular differences between SUSY signals and the scalar ones are even more reduced when are added the decay products of their respective new states. Therefore, we expect similar efficiencies between the signals of our model and those coming from SUSY, allowing us to recast LEP-II bounds.

In Fig. 1(a) we show the recast limits from neutralinos searches at LEP-II to our model [50]. The allowed region is a small, narrow, blue area close to the LEP energy threshold. The exclusion region is notoriously higher than the scalar case [48] because the production cross section for vectors present an enhancement through their longitudinal polarization, compared to the scalar case [see (9)]. The resulting excluded region shown in Fig. 1(a) can be expressed as

$$
\begin{aligned}
& M_{V^{1}}<100 \mathrm{GeV} \text { and } M_{V^{2}}<200 \mathrm{GeV} \text { and } \\
& M_{V^{2}}-M_{V^{1}}>8 \mathrm{GeV},
\end{aligned}
$$

and $M_{V 1}+M_{V 2}$ must to be a few GeV less than the LEP threshold $(189 \mathrm{GeV})$. Only the white region in which the neutral vector states are near the degeneracy is not explored by LEP-II.

Additionally, charginos searches [51] also put strong constraints on the charged vector states $V^{ \pm}$. As it is shown in Fig. 1(b), the excluded region on the charged vectorial mass results to be

$$
M_{V^{ \pm}} \lesssim 93 \mathrm{GeV}
$$

\section{B. $H \rightarrow \gamma \gamma$ constraints from $\mathrm{LHC}$ data}

In the SM, there is no interaction between the Higgs and photons at tree level. However, the Higgs boson can decay into a pair of photons due to one-loop processes in which the charged gauge bosons $W^{ \pm}$and fermions run in the internal lines. In our model the new doublet vector plays an important role introducing new corrections to $\Gamma(H \rightarrow \gamma \gamma)$ through the running of charged vectors $V^{ \pm}$in the internal lines at one-loop. Recent measurements show that the experimental value of $\Gamma(H \rightarrow \gamma \gamma)$ is very close to the SM prediction, implying strong restrictions for models that go beyond the SM [52].

The partial decay width of the Higgs boson into two photons in the DVDM is

$$
\begin{aligned}
\Gamma(H \rightarrow \gamma \gamma)= & \frac{\alpha_{e m} M_{H}^{3}}{256 \pi^{3} v^{2}} \mid \sum_{i} N_{c i} Q_{i}^{2} F_{1 / 2}\left(\beta_{i}\right) \\
& +F_{1}\left(\beta_{W}\right)+\left.\frac{\lambda_{2}}{2}\left(\frac{v}{M_{V^{ \pm}}}\right)^{2} F_{1}\left(\beta_{V}\right)\right|^{2},
\end{aligned}
$$




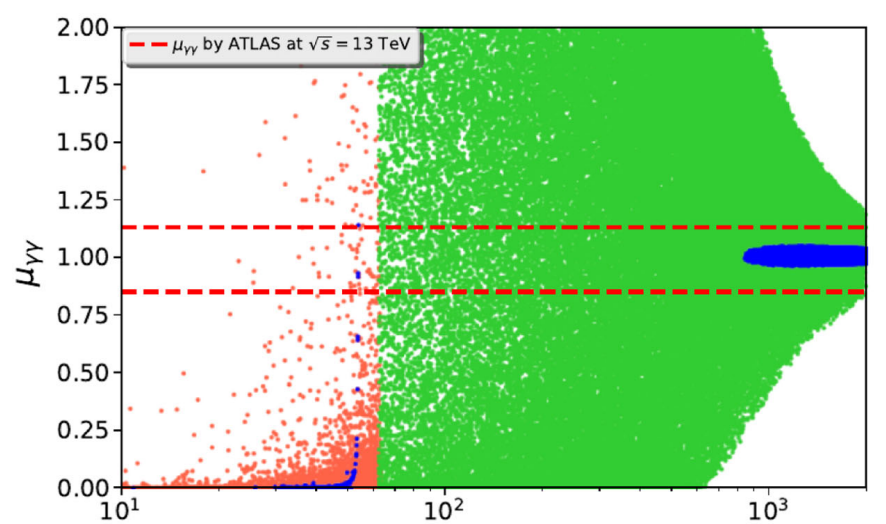

(a) $\mathrm{Mv}_{1}(\mathrm{GeV})$

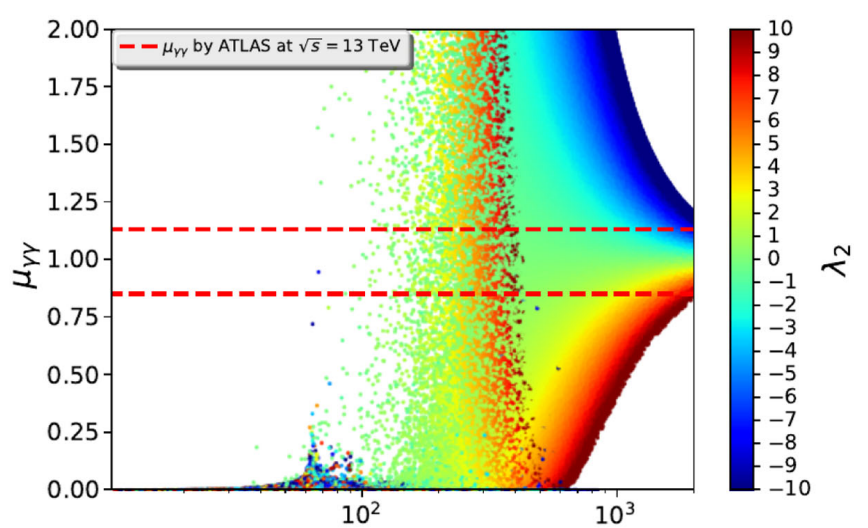

(b) $M_{V^{ \pm}}(\mathrm{GeV})$

FIG. 2. (a) Diphoton rate $\mu^{\gamma \gamma}$ vs DM mass $M_{V^{1}}$ in two regions: the pink region correspond to $M_{V^{1}} \leq M_{H} / 2$, where $H \rightarrow V^{1} V^{1}$ channel is open, and the green one to $M_{V^{1}}>M_{H} / 2$, where $H \rightarrow V^{1} V^{1}$ channel is closed. The blue color represents relic saturation. (b) Color map for diphoton rate as a function of the parameter $\lambda_{2}$ and the charged vector mass $M_{V^{ \pm}}$. For these pictures we took into account perturbability restrictions (12). The horizontal red lines represent the global signal strength coming from $\sqrt{s}=13$ TeV ATLAS Higgs data analysis (18).

where $\alpha_{e m}$ is the electromagnetic fine-structure constant, $M_{H}$ is the mass of the Higgs boson, $v$ is the $245 \mathrm{GeV}$ Higgs field vacuum expectation value (vev), $N_{c i}, Q_{i}$, and $\beta_{i}=$ $4 M_{i}^{2} / M_{H}^{2}$ are the color factor, the electric charge, and a dimensionless factor respectively for a certain $i$ fermion running in the loop. In the same way we define the dimensionless factors $\beta_{W}=4 M_{W}^{2} / M_{H}^{2}$ and $\beta_{V}=$ $4 M_{V}^{2} / M_{H}^{2}$ for the charged $W^{ \pm}$boson and the new charged vector $V^{ \pm}$contributions in the loop, respectively. The functions $F_{i}$ are loop factors for particles of spin given in the subscript:

$$
\begin{aligned}
F_{1 / 2} & =-2 \beta(1+(1-\beta) f(\beta)), \\
F_{1} & =2+3 \beta+3 \beta(2-\beta) f(\beta),
\end{aligned}
$$

with

$$
f(\beta)= \begin{cases}\arcsin (1 / \beta)^{2}, & \text { for } \beta \geq 1 \\ -\frac{1}{4}\left[\ln \frac{1+\sqrt{1-\beta}}{1-\sqrt{1-\beta}}-i \pi\right]^{2}, & \text { for } \beta<1 .\end{cases}
$$

We consider the most recent limit coming from the $\sqrt{s}=$ $13 \mathrm{TeV}$ ATLAS Higgs data analysis [52] to set restrictions on the parameter space. The new contributions respect to the SM are parametrized as the ratio of the branching ratios between our model and the SM

$$
\frac{\mathrm{Br}^{B S M}(H \rightarrow \gamma \gamma)}{\operatorname{Br}^{\mathrm{SM}}(H \rightarrow \gamma \gamma)} \equiv \mu^{\gamma \gamma}=0.99 \pm 0.14
$$

The new contributions to $\mu^{\gamma \gamma}$ are governed by the parameters $\lambda_{2}$ and $M_{V^{ \pm}}$or, equivalently, by $\lambda_{L}$ and the difference of masses between $M_{V^{1}}$ and $M_{V^{ \pm}}$, as previously shown in Eq. (9).
In Fig. 2(a) we present the diphoton rate as a function of the DM mass $M_{V^{1}}$ where the parameter space was divided in two regions: the pink points $\left(10 \leq M_{V^{1}} \leq M_{H} / 2\right)$ represent the zone where the decay mode $H \rightarrow V^{1} V^{1}$ is open making the decay mode $H \rightarrow \gamma \gamma$ very low and therefore pushing the $\mu^{\gamma \gamma}$ under the experimental limit for most of the points, and the green points $\left(M_{V^{1}}>M_{H} / 2\right)$ represent the zone where the decay mode $H \rightarrow V^{1} V^{1}$ is closed. In both regions we show in blue the points which are consistent with the observed amount of DM. In Fig. 2(b) we present a color map of the parameter $\lambda_{2}$ as a function of the diphoton rate vs charged vector mass $M_{V^{ \pm}}$. In both cases the horizontal red lines represent the global signal strength coming from $\sqrt{s}=13$ TeV ATLAS Higgs data analysis (18).

We can notice that diphoton rate constraints are very restrictive ruling out an important amount of the parameter space, mostly when $\left|\lambda_{2}\right|$ takes big values in the region $M_{V^{ \pm}} \gtrsim 400 \mathrm{GeV}$. However, for higher masses such as $M_{V^{ \pm}} \gtrsim 1 \mathrm{TeV}$, still there is a region where $\mu^{\gamma \gamma}$ is within the experimental limit for high couplings, e.g., $\left|\lambda_{2}\right|>5$. Another interesting feature of the constraint is that the low mass region that can satisfied the Planck limit for $\Omega_{\mathrm{DM}} h^{2}$ is practically ruled out. Finally, the high mass region which saturates the Planck limit matches perfectly with the $\mu^{\gamma \gamma}$ measurements where $\left(\left|\lambda_{2}\right|<2\right)$ and $\left(M_{V^{ \pm}}-M_{V^{1}} \lesssim 20 \mathrm{GeV}\right)$ values are preferred.

\section{Invisible Higgs decay from LHC data}

The Higgs boson is one of the portals connecting the dark sector with the SM, however there is an important restriction that we need to worry about. When $M_{V^{1}} \leq M_{H} / 2$, the SM-Higgs boson can decay into dark matter particles, which translate into invisible decays. On the other hand, both ATLAS and CMS experiments at the 
LHC have been searching for Higgs invisible decays at $\sqrt{s}=7,8$ and $13 \mathrm{TeV}$, putting the restrictive upper limit

$$
\operatorname{Br}(H \rightarrow \text { inv })<24 \%,
$$

at a $95 \%$ of confidence level $[53,54]$. In this section we interpret the CMS upper bound as the maximum possible branching ratio of the Higgs boson into dark matter particles, i.e.,

$\operatorname{Br}\left(H \rightarrow V^{1} V^{1}\right) \equiv \frac{\Gamma\left(H \rightarrow V^{1} V^{1}\right)}{\Gamma_{\mathrm{SM}}+\Gamma\left(H \rightarrow V^{1} V^{1}\right)}<\mathrm{Br}_{\text {inv }}^{\max }$,

where $\mathrm{Br}_{\text {inv }}^{\max }=24 \%$, and $\Gamma_{\mathrm{SM}}$ corresponds to the full decay width of the SM Higgs. In our model, the decay width of the Higgs to two dark matter particles is given by

$$
\begin{aligned}
\Gamma\left(H \rightarrow V_{1} V_{1}\right)= & \frac{M_{W}^{2} \lambda_{L}^{2}}{8 \pi g^{2} M_{H}}\left(3-\frac{M_{H}^{2}}{M_{V^{1}}^{2}}+\frac{1}{4} \frac{M_{H}^{4}}{M_{V^{1}}^{4}}\right) \\
& \times \sqrt{1-\frac{4 M_{V^{1}}^{2}}{M_{H}^{2}}}
\end{aligned}
$$

where $g$ is the weak coupling constant. Replacing (21) into (20) and solving for $\lambda_{L}$, we found the following constraint

$$
\left|\lambda_{L}\right|<\left(\frac{8 \pi \Gamma_{\mathrm{SM}} g^{2} M_{H}\left(\frac{1}{\mathrm{Br}_{\mathrm{inv}}^{\max }}-1\right)^{-1}}{M_{W}^{2}\left(3-\frac{M_{H}^{2}}{M_{V^{1}}^{2}}+\frac{1}{4} \frac{M_{H}^{4}}{M_{V^{1}}^{4}}\right) \sqrt{1-4 M_{V^{1}}^{2} / M_{H}^{2}}}\right)^{1 / 2} .
$$

This bound is extremely restrictive because it allows only for very small values of $\lambda_{L}{ }^{2}$ For example, when $M_{V^{1}}$ is close to $M_{H} / 2(\sim 60 \mathrm{GeV})$, relation (22) sets $\lambda_{L} \lesssim 0.03$. This constraints is complementary to the one given by Higgs diphoton decay, which strongly constrained dark matter masses below $M_{H} / 2$, eliminating almost completely the region $M_{V^{1}} \leq M_{H} / 2$.

The case described above was based on the assumption that the sole channel contributing to the Higgs invisible decay is $H \rightarrow V^{1} V^{1}$. However, when $M_{V^{2}}<M_{H} / 2$, the channel $H \rightarrow V^{2} V^{2}$ can also contribute to the invisible Higgs decay provided that $\Delta M=M_{V^{2}}-M_{V^{1}}$ is small enough (of the order of a few $\mathrm{GeV}$ or less), to forbid $V^{2}$ to decay into $V^{1}$ and a detectable pair of fermions. Considering that $\lambda_{R}=\lambda_{L}+\frac{2\left(M_{V^{2}}^{2}-M_{V^{1}}^{2}\right)}{v^{2}}$, and in this case, $M_{V^{2}} \approx M_{V^{1}}$, then $\lambda_{L} \approx \lambda_{R}$. Therefore, in this case the limit on $\lambda_{L}$ can be easily modified.

Finally, in the case of a small $V^{ \pm}-V^{1}$ mass split, the channel $H \rightarrow V^{ \pm} V^{\mp}$ may also contributes to the Higgs

\footnotetext{
${ }^{2}$ This strong constraint in the coupling among the Higgs boson and the dark matter is also shown in the i2HDM [55] with similar results.
}

invisible decay channel. However, LEP limits (15) put very strong constraints on the allowed masses of the charged vectors, then making the Higgs decay into the on-shell charged vectors kinematically forbidden.

\section{Relic density constraints}

As we mentioned in Sec. III, our model has a 6-dimensional parameter space but only four free parameters are relevant for our study: three physical masses of the vector fields $\left(M_{V^{1}}, M_{V^{2}}, M_{V^{ \pm}}\right)$, and one coupling constant $\left(\lambda_{L}\right)$ between the SM-Higgs boson and $V^{1}$. In order to show a general qualitative description of the dark matter relic density $\Omega_{\mathrm{DM}} h^{2}$ as a function of the parameter space, we can fix some of them and perform a scan over the more relevant ones. The result should be in agreement with Planck [56] measurements:

$$
\Omega_{\mathrm{DM}} h^{2}=0.1184 \pm 0.0012
$$

The interaction between both the dark sector and the SM is through the SM-Higgs boson and the electroweak gauge bosons, however the interaction with the latter it is fixed by gauge couplings. For simplicity we will consider as well $M_{V^{2}}=M_{V^{ \pm}}{ }^{3}$. Therefore the two relevant parameters are $\left(M_{V^{1}}, \lambda_{L}\right)$.

We will present two characteristic scenarios which we will refer to as: (a) quasidegenerate case, where $\Delta M=M_{V^{2}}-M_{V^{1}}=1 \mathrm{GeV}$, and (b) the nondegenerate one, in which $\Delta M=M_{V^{2}}-M_{V^{1}}=100 \mathrm{GeV}$. In Fig. 3 we present a 2-dimensional parameter space where we show the $\Omega_{\mathrm{DM}} h^{2}$ as a function of the DM mass $M_{V^{1}}$ for different values of $\lambda_{L}$ in the two scenarios mentioned above. The horizontal red dashed line corresponds to the central value of the relic density measured by Planck.

The first important aspect we can appreciate of this model is that there are two regions in which it can fulfill the DM budget. The first saturation zone happens between $30<M_{V^{1}}<80 \mathrm{GeV}$ for a nondegenerate scenario, as we can see from Fig. 3(b). In this case the main mechanism of annihilation is through s-channel Higgs boson exchange which is controlled by the $\lambda_{L}$ coupling. Interestingly, there is a considerable area of overabundance for small values of $M_{V^{1}}$ even for large values of $\lambda_{L}$. Of course, this region must be excluded as nonphysical.

The second saturation region takes place when $M_{V^{1}}>$ $830 \mathrm{GeV}$ in the quasidegenerate scenario [see Fig. 3(a)]. In this zone the interaction between the DM and the longitudinal polarization of $W^{ \pm}$and $Z$ boson becomes dominant. This interaction is modulated by $\lambda_{i}$ quartic couplings which in turn depend on the mass difference among the new vectors as it is shown in Eq. (9). When $\Delta M$ is small the $\lambda_{i}$ become small enough to produce a suppression in the

\footnotetext{
${ }^{3}$ This equivalently to do $\lambda_{3}=\lambda_{4}$ as you can easily check from (7).
} 

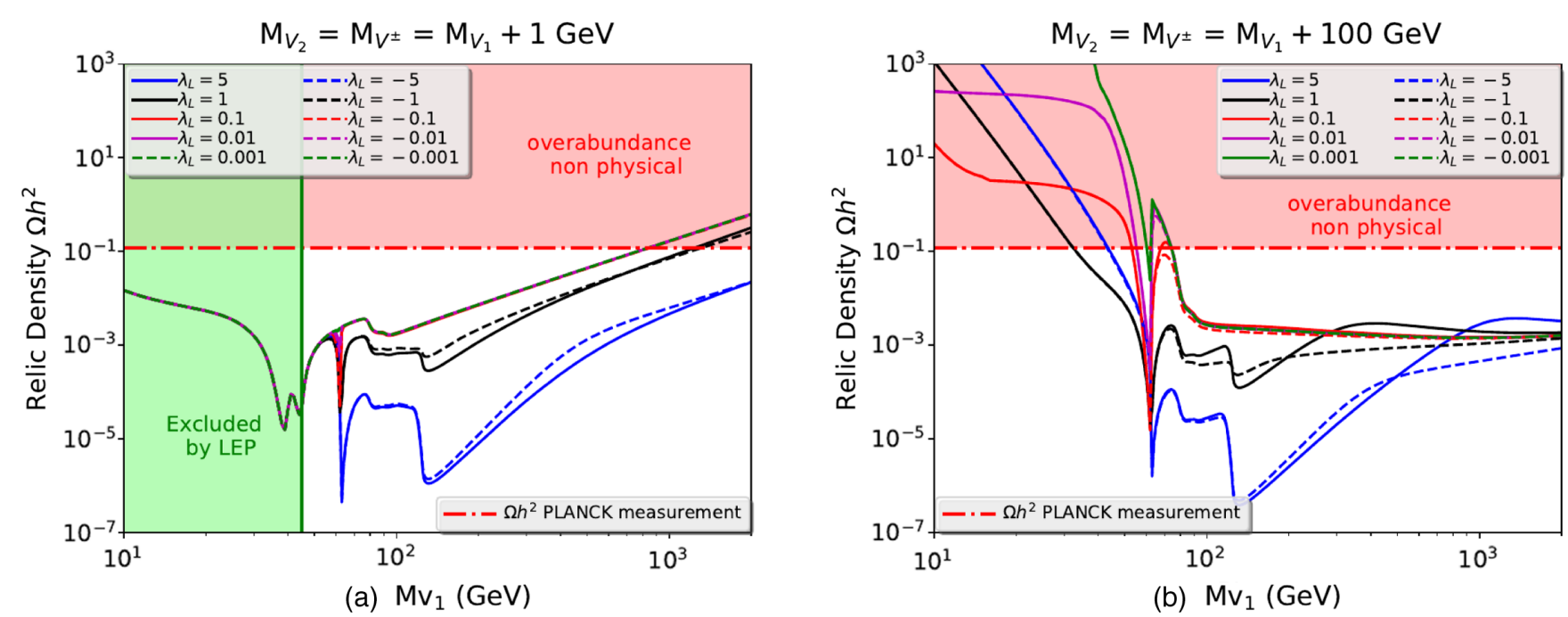

FIG. 3. Relic density $\Omega_{\mathrm{DM}} h^{2}$, as a function of $M_{V^{1}}$ for different values of $\lambda_{L}$ in a quasidegenerate scenario (a) where $M_{V^{2}}=M_{V^{ \pm}}=$ $M_{V^{1}}+1 \mathrm{GeV}$ and a nondegenerate scenario (b) where $M_{V^{2}}=M_{V^{ \pm}}=M_{V^{1}}+100 \mathrm{GeV}$. The horizontal red line corresponds to the central value of the relic density measured by Planck. The green area indicates the excluded region by LEP measurements.

annihilation average cross section for these channels pushing the DM abundance up to reach the saturation limit, even when the (co)annihilation effects are present which become subdominant. In contrast, in the nondegenerate cases the annihilation of DM is more efficient due to the large values of $\lambda_{i}$ which results in the asymptotically flat behavior of abundance for high DM mass values.

The overabundance seen in the nondegenerate scenario for small values of $M_{V^{1}}$ completely disappears in the quasidegenerate case due to the effects of (co)annihilation which introduces new sources of annihilation of DM, pushing the abundance below the Planck experimental limit. When $M_{V^{1}} \sim 40$ and $M_{V^{1}} \sim 45 \mathrm{GeV}$ we can note the effects of resonant (co)annihilation through $V^{1} V^{ \pm} \rightarrow$ $W^{ \pm}$and $V^{1} V^{2} \rightarrow Z$ channels, respectively, that manifest on the Fig. 3(a) as two inverted peaks.

At exactly $M_{V^{1}} \sim 62.5 \mathrm{GeV}$ the resonant annihilation through the Higgs boson take place as we can see in both scenarios as a deep peak. After that resonance we observe three points where the abundance of DM decreases considerably. This happens markedly at $M_{V^{1}} \sim 80 \mathrm{GeV}$ through the opening channel $V^{1} V^{1} \rightarrow W^{+} W^{-}$and more tenuously at $M_{V^{1}} \sim 90 \mathrm{GeV}$ through $V^{1} V^{1} \rightarrow Z Z$. Finally at $M_{V^{1}} \sim 125 \mathrm{GeV}$ the opening of $V^{1} V^{1} \rightarrow H H$ take place corresponding to the reduction of DM relic density through s-channel Higgs boson.

One can also observe that in the case of $\Delta M=$ $100 \mathrm{GeV}$, for $M_{V^{1}}$ below $65 \mathrm{GeV}$, DM coannihilation is suppressed and the relic density is equal or below the experimental limit only for large values of $\lambda_{L}\left(\lambda_{L}>0.1\right)$ which are excluded by LHC limits on the invisible Higgs decay.

Finally, it is easy to notice that for larger values of $\lambda_{L}$ the abundance of DM decreases, however, is important to stress that there is a slight difference for the case in which $\lambda_{L}$ takes positives and negatives values after $M_{V^{1}} \sim 62.5 \mathrm{GeV}$. This behavior is due the interference effect between the schannel Higgs boson exchange diagram and those involving gauge bosons.

\section{E. Direct detection limits}

We consider as well whether our model is consistent with limits coming from XENON1T [57] experiment studying the rescaled spin independent proton-DM scattering cross section

$$
\hat{\sigma}_{\mathrm{SI}}=\left(\Omega_{\mathrm{DM}} / \Omega_{\text {Planck }}\right) \times \sigma_{\mathrm{SI}}\left(V^{1} p \rightarrow V^{1} p\right)
$$

which allows us to take into account the case when the vector $V_{1}$ contribute only partially to the total amount of DM. This approach is useful to take into account other sources that can contribute to fulfill the DM budget. We present the $\hat{\sigma}_{S I}$ as a function of the DM mass for several values of $\lambda_{L}$ in the quasidegenerate and nondegenerated scenario as we shown in Fig. 4. The green area, shown in both plots is the excluded region from the direct detection (DD) experiment and the soft red color in Fig. 4(a) is excluded by LEP data.

The $\sigma_{\mathrm{SI}}$ is through the t-channel with the Higgs boson as a mediator, therefore we can notice immediately that $\lambda_{L}$ plays an important roll which is scale the strength of the interaction between DM and nucleus of ordinary matter. In the quasidegenerated scenario the asymptotically flat behavior of the $\hat{\sigma}_{\mathrm{SI}}$ for $M_{V^{1}}>100 \mathrm{GeV}$ can be explained because as $M_{V^{1}}$ take higher values, the cross section $\sigma_{\mathrm{SI}}$ is decreasing, however this effect is compensated by the fact that there is more abundance of DM as the value of $M_{V^{1}}$ is increasing. We can check this from Fig. 3(a). On the other 


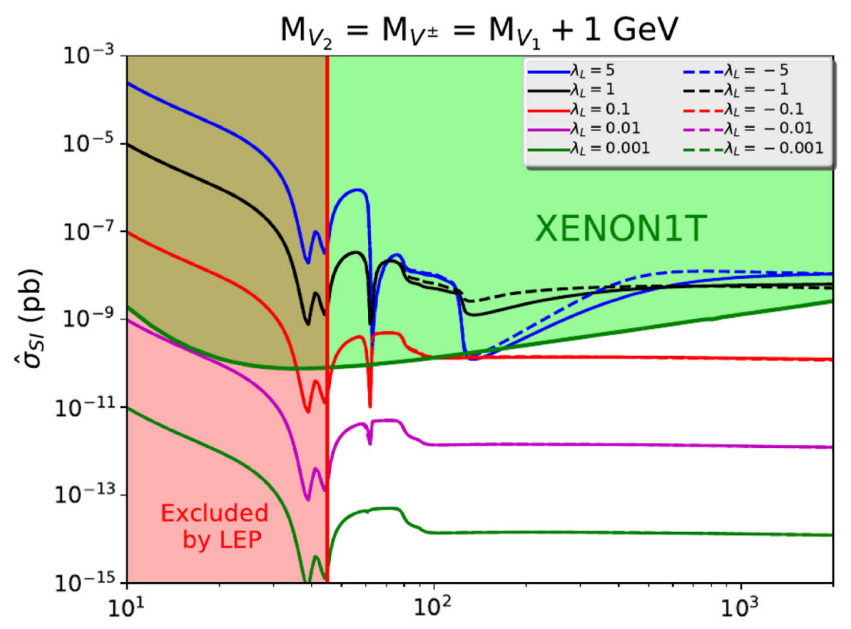

(a) $\mathrm{Mv}_{1}(\mathrm{GeV})$

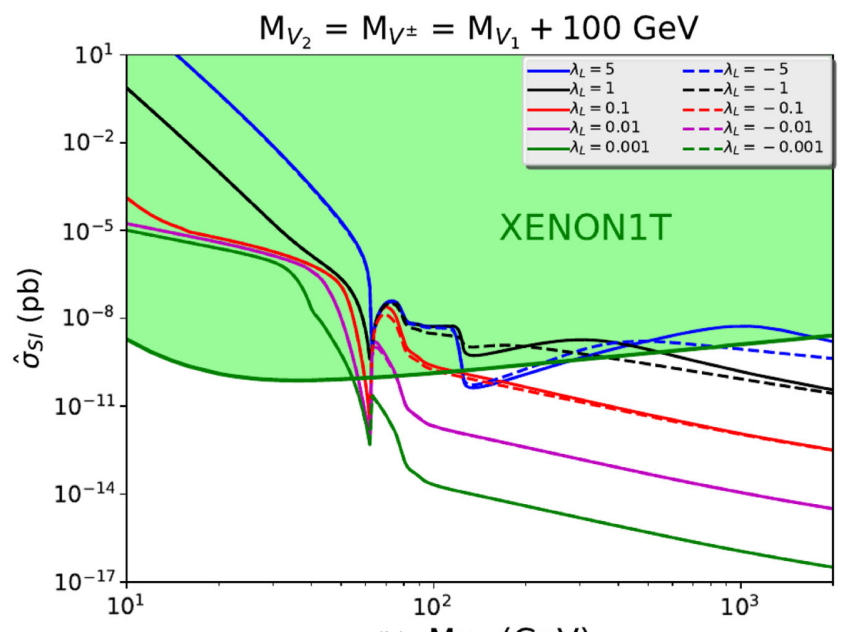

(b) $M v_{1}(\mathrm{GeV})$

FIG. 4. Rescaled spin independent direct detection cross section $\hat{\sigma}_{\mathrm{SI}}$ versus $M_{V^{1}}$ and the XENONT1 constraint for several values of $\lambda_{L}$. The red-shaded region in the left frame is excluded by LEP data.

hand, in the nondegenerate scenario the $\Omega_{\mathrm{DM}} h^{2}$ is relatively constant after the DM annihilation channel $V^{1} V^{1} \rightarrow H H$ is opened [Fig. 3(b)], therefore, as the value of DM mass is increasing the $\hat{\sigma}_{\mathrm{SI}}$ is taking smaller values.

\section{F. Indirect detection limits}

For indirect detection (ID) constraints, we distinguish two dark matter mass regions. The first one corresponds to the low mass regime, $M_{\mathrm{DM}}<M_{W}$, which is governed by the dark matter annihilation to fermions through a virtual Higgs in the s-channel, mostly to $V^{1} V^{1} \rightarrow b \bar{b}$. However, considering that LEP, LHC and direct detection constraints ruled out dark matter masses in this region, we do not take into account constraints coming from ID in this case.

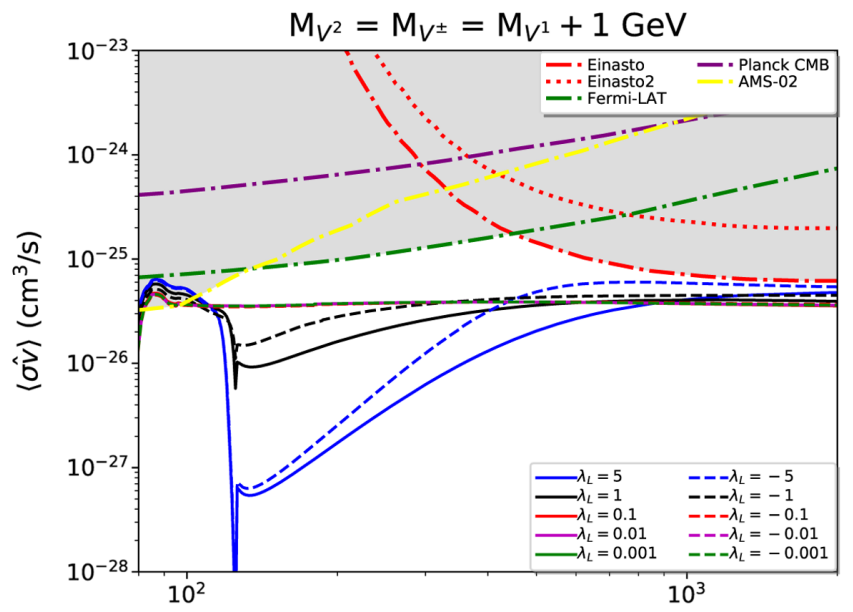

(a) $M v_{1}(\mathrm{GeV})$
The second region corresponds to both intermediate and high mass regime of the model, $M_{V^{1}}>M_{W}$, in which the dark matter particles annihilates mostly in the channels $V^{1} V^{1} \rightarrow W^{+} W^{-}, Z Z, H H, t \bar{t}$. Hadronization of weak gauge bosons and Higgs boson produces neutral pions, which in turn decay into photons generating a gamma-ray flux with a featureless spectrum which could be detected at Earth. We exemplify the ID constraints for the channel $V^{1} V^{1} \rightarrow W^{+} W^{-}$, for being one with the bigger cross section in the region of interest. Considering the rescaled average annihilation cross section

$$
\langle\hat{\sigma v}\rangle=\left(\Omega_{\mathrm{DM}} / \Omega_{\text {Planck }}\right) \times\langle\sigma v\rangle,
$$

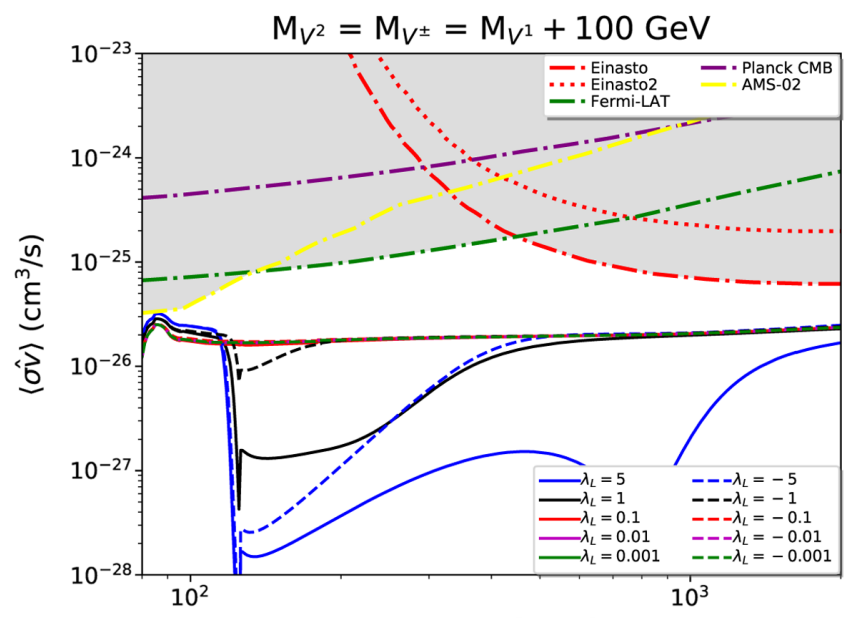

(b) $M v_{1}(\mathrm{GeV})$

FIG. 5. Rescaled average annihilation cross section versus $M_{V^{1}}$ for $V^{1} V^{1} \rightarrow W^{+} W^{-}$channel. The scan was done for different values of $\lambda_{L}$ for the quasidegenerate (a) and nondegenerate (b) case. We considered limits coming from HESS (Einasto and Einasto2), Fermi-LAT, AMS-02, and Planck. The gray region above shows the exclusion given by the experiments mentioned. 
when the DM relic density is less than that measured by Planck, in Fig. 5 we show the predictions from our model using MICROMEGAs in both cases the quasidegenerate scenario (a) and the nondegenerate (b).

In Fig. 5 we take into account upper limits data from gamma-ray experiments such as HESS [58] and Fermi-Lat [59], antiprotons measurements made by AMS-02 [60], and constraints from temperature anisotropies of the CMB by Planck satellite [61]. In the low mass region where $M_{V^{1}} \approx M_{W}$, AMS-02 and Fermi-Lat experiments are the most sensitive to the annihilation cross section predicted by the model. However, the exclusion is very tiny in comparison to the other constraints coming from diphoton rate or direct detection. For higher dark matter masses, none of the experiments are sensitive enough to explore the predictions on $\langle\sigma v\rangle$. However, it seems that HESS will be able soon to prove the predictions of our model at the $\mathrm{TeV}$ mass scale. Therefore, ID does not put any significant constraint on the model until now.

\section{DARK MATTER PHENOMENOLOGY}

The previous description provides us with a qualitative overview of the parameter space. However, in order to have a deeper understanding of the model we perform a random scan using 7 million points of the most relevant parameters that have direct interference in the phenomenology of dark matter. The range of the parameters used in the scan can be summarized in Table I.

The result of our scan is presented in Fig. 6 where we show several plots with 2-D projections of the 4dimensional parameter space as a color map of DM relic density. We considered the parameter space without any theoretical or experimental constraint in the first row, and then, in the second row we took into account perturbativity (12), LEP limits (13), (14), and (15), Higgs decay into two

TABLE I. Range of the 4-dimensional parameter space.

\begin{tabular}{lcc}
\hline \hline Parameter & Min value & Max value \\
\hline$M_{V^{1}}[\mathrm{GeV}]$ & 10 & 2000 \\
$M_{V^{2}}[\mathrm{GeV}]$ & 10 & 2000 \\
$M_{V^{ \pm}}[\mathrm{GeV}]$ & 10 & 2000 \\
$\lambda_{L}$ & -12 & 12 \\
\hline \hline
\end{tabular}

photons (18), invisible Higgs decay (19), overabundance DM relic density (23) Xenon1T direct detection and finally HESS, Fermi-Lat, AMS-02, and CMB indirect detection constraints.

As we explained previously, and without losing generality, we work in the region where $M_{V^{1}}<M_{V^{2}}$ and therefore $\lambda_{4}>0$. For this reason we exclude the region $M_{V^{1}}>M_{V^{2}}$ as we can see from the gray region in Fig. 6(b).

The different pattern of colors represents the amount of DM that the model is capable of explaining considering a thermal production mechanism, where the dark red color in the low DM mass region $\left(M_{V^{1}} \lesssim 45 \mathrm{GeV}\right)$ of Fig. $6(\mathrm{a}, \mathrm{b})$ represents overabundance which we consider as nonphysical. The dark blue colors are the regions with extreme underabundance of DM, which is more accentuated for large values of $\lambda_{L}$ in the zone where $M_{V^{1}}>M_{H} / 2$ after the respective annihilation channels $(W W, Z Z$ and $H H)$ are progressively opened, reflecting the same pattern shown previously in Fig. 3.

Looking at Fig. 6(a,b), the resonant annihilation through the Higgs boson is easily recognized by the vertical separation around $M_{V^{1}} \sim 62.5 \mathrm{GeV}$ where a steep break in the color pattern can be seen, changing from a light green to a dark blue. We can also notice the resonant (co)annihilation through the $Z$ boson in the plane $\left(M_{V^{1}}, M_{V^{2}}\right)$ of Fig. 6(b) at the region $M_{V^{1}}=M_{V^{2}} \sim 45 \mathrm{GeV}$.

Taking into account perturbative restrictions, the region of the parameter space that shows an important mass difference between $M_{V^{1}}$ and $M_{V^{2}}$ is excluded since this large difference increases the values of the quartic coupling beyond the allowed value set by (12). This effect can be seen clearly in Fig. 6(d) where the region with $M_{V^{2}}>$ $900 \mathrm{GeV}$ for $M_{V^{1}}<500 \mathrm{GeV}$ is excluded. Only when the mass difference becomes relatively small, $M_{V^{2}}$ can admit larger values.

By incorporating the restrictions coming from Higgs invisible decay almost all the parameter space for $M_{V^{1}} \lesssim$ $M_{H} / 2$ disappears with exception of a very narrow region where $\lambda_{L}$ parameter take small values $\left(\lambda_{L} \lesssim 0.02\right)$. This happens because the dominant annihilation channel is through the Higgs boson exchange.

The Higgs diphoton rate (18) introduces strong restrictions on the parameter space especially for negative values of $\lambda_{L}$. We can see that restriction in the Fig. 6(c) where $\lambda_{L}$ is

TABLE II. Total cross section (fb) for $p p \rightarrow X+E,(X=j, Z, H)$ with $\lambda_{345}\left(\lambda_{L}\right)=0.1$, NNPDF23_10_ as_0130_qed (proton) as a PDF, and $p_{T}^{X}>100 \mathrm{GeV}$. Here, the missing energy is due to the production of $V_{1} \bar{V}_{1}, V_{1} \bar{V}_{2}$, and $V_{2} V_{2}$. The same for the scalar case.

\begin{tabular}{|c|c|c|c|c|c|c|}
\hline \multirow{2}{*}{$\begin{array}{l}\text { Model } \\
\text { Mass (GeV) }\end{array}$} & \multicolumn{3}{|c|}{ i2HDM } & \multicolumn{3}{|c|}{ DVDM } \\
\hline & 100 & 500 & 800 & 100 & 500 & 800 \\
\hline Mono- $j$ & $1.9 \times 10^{1}$ & $6.6 \times 10^{-2}$ & $6.4 \times 10^{-3}$ & $7.3 \times 10^{3}$ & 6.2 & $4.5 \times 10^{-1}$ \\
\hline Mono-Z & $3.7 \times 10^{-1}$ & $2.9 \times 10^{-3}$ & $3.1 \times 10^{-4}$ & $7.3 \times 10^{2}$ & $4.1 \times 10^{-1}$ & $2.8 \times 10^{-2}$ \\
\hline Mono- $H$ & $1.0 \times 10^{-2}$ & $2.0 \times 10^{-5}$ & $2.2 \times 10^{-6}$ & $3.0 \times 10^{2}$ & $3.1 \times 10^{-3}$ & $9.7 \times 10^{-6}$ \\
\hline
\end{tabular}




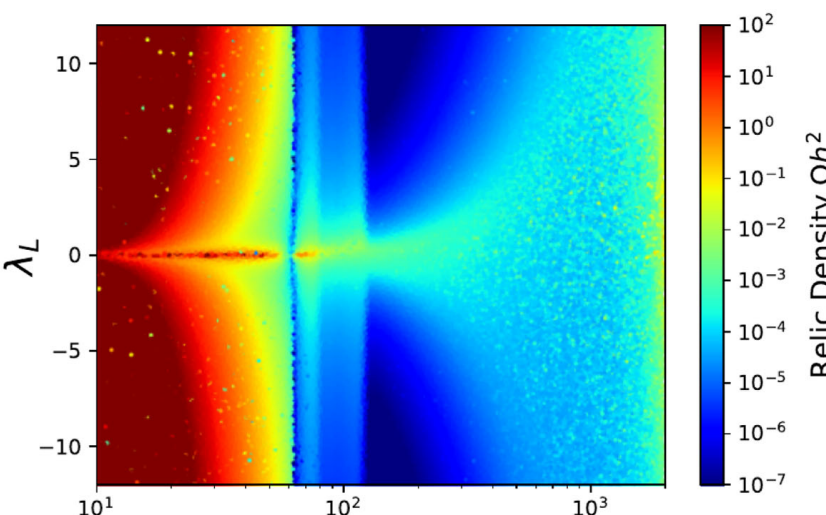

(a) $M v_{1}(\mathrm{GeV})$

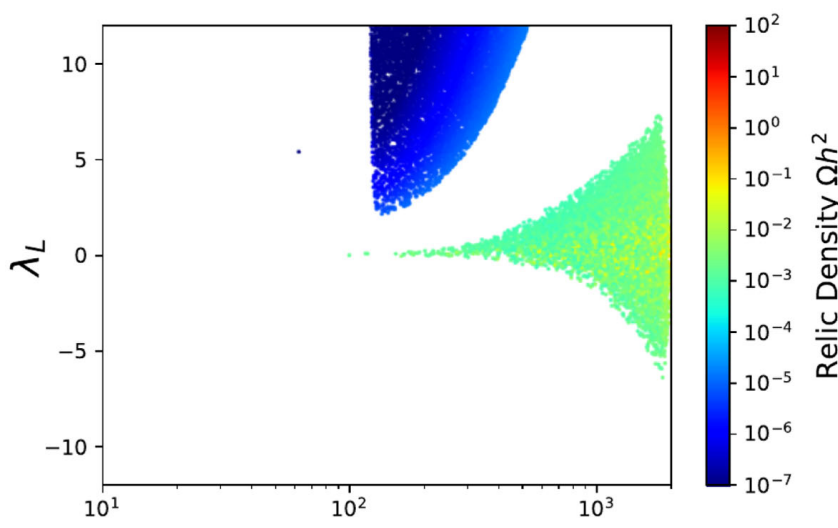

(c) $M v_{1}(\mathrm{GeV})$

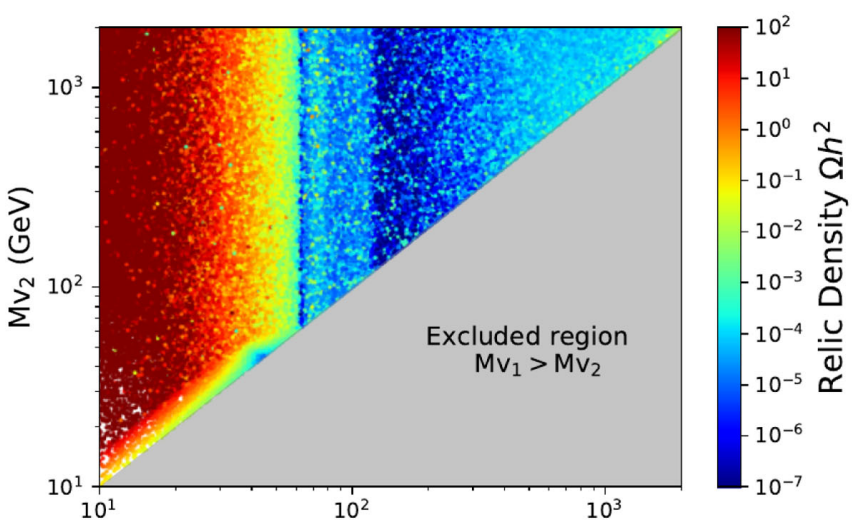

(b) $M v_{1}(\mathrm{GeV})$

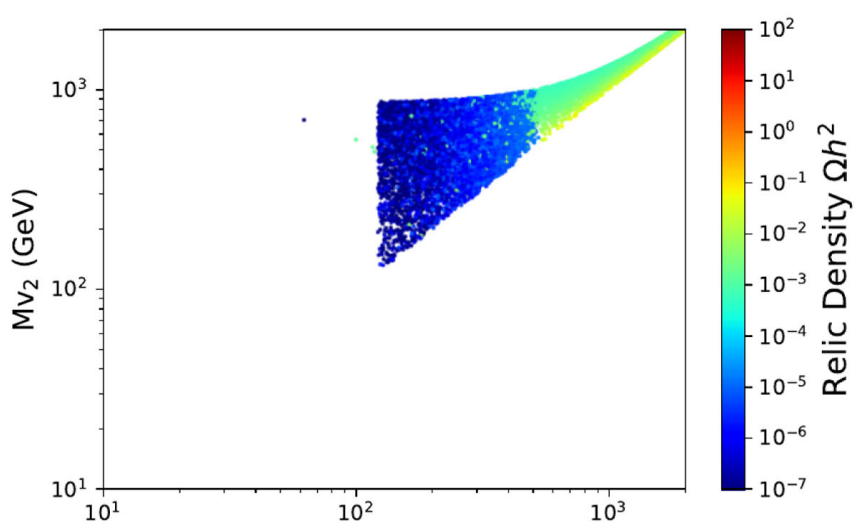

(d) $M v_{1}(\mathrm{GeV})$

FIG. 6. 2-D projections of the 4-dimensional parameter space presented as a color map of $\Omega_{\mathrm{DM}} h^{2}$ in two different planes: $\left(M_{V^{1}}, \lambda_{L}\right)$ plane for Fig. (a,c) and $\left(M_{V^{1}}, M_{V^{2}}\right)$ plane for Fig. (b,d). In the first row we present the parameter space without any constraint and in the second one we applied all the theoretical and experimental constraints with the exception of DM underabundance.

limited from below through the parabolic shape as we increase the values of $M_{V^{1}}$. The diphoton rate depend explicitly on $\lambda_{2}=\lambda_{L}+2\left(M_{V^{1}}^{2}-M_{V^{ \pm}}^{2}\right) / v^{2}$, where the difference of squared masses is always negative because $M_{V^{1}}<M_{V^{ \pm}}$, therefore when the mass difference is large and $\lambda_{L}$ takes high negative values, the parameter $\lambda_{2}$ grows in demacy, causing a great deviation from the experimental value of $\mu^{\gamma \gamma}$, this can also be seen as well in Fig. 2(b).

The additional constraint from XENON1T DD experiments removes part of the parameter space contained between $63<M_{V^{1}}<125 \mathrm{GeV}$ where the direct detection rate is more sensitive. This affects the region for positive and negative values of $\lambda_{L}$, however the negative part was removed previously by the Higgs diphoton rate constraint as we can see from Fig. 6(c). The scattering cross section between $V_{1}$ and nuclei is through the t-channel with the Higgs boson as a mediator, therefore it depends explicitly on the parameter $\lambda_{L}$. For large values of $\lambda_{L}$ the abundance of DM is low, but not low enough to suppress the DM detection rate through DD signal. Only when $\lambda_{L}$ is small $(\lesssim 0.02)$, the region between $90<M_{V^{1}}<200 \mathrm{GeV}$ of the parameter space is able to bypass the limits of direct detection. When we move to a high DM mass region $\left(M_{V^{1}} \gtrsim 200 \mathrm{GeV}\right)$, where the DD rate is less sensitive, we still have an excluded region with parabolic shape that is only reached for large values of $\lambda_{L}$. It produces a clear division between a low density of DM zone with the rest of the parameter space. However, in the case of high degeneracy among the vector masses for the region $M_{V^{1}}>$ $900 \mathrm{GeV}$ the DD rate is able to restrict parameter space for values of $\lambda_{L}$ up to 1 , as we will see later in the next subsection.

\section{A. Vector dark matter as the only source}

In the previous paragraphs, we considered experimental and theoretical constraints in our parameter space but we maintained the assumption that our DM candidate contributes partially to the DM budget, therefore we relaxed the lower limit on the relic density give by Planck. Here, we show how the model can completely explain the abundance of DM for some special region of the parameter space taking into account both upper and lower Planck limits at $1 \sigma$ (23). For that reason, in Fig. 7 we present a 2D 


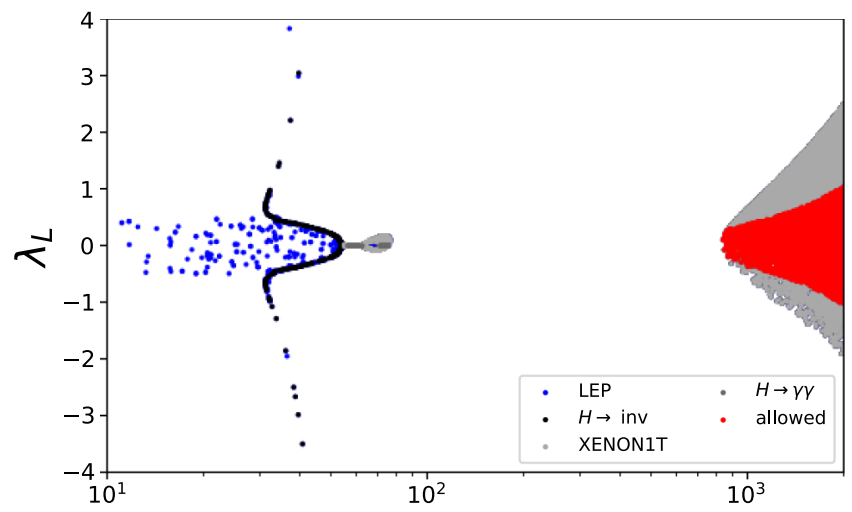

(a) $M v_{1}(\mathrm{GeV})$

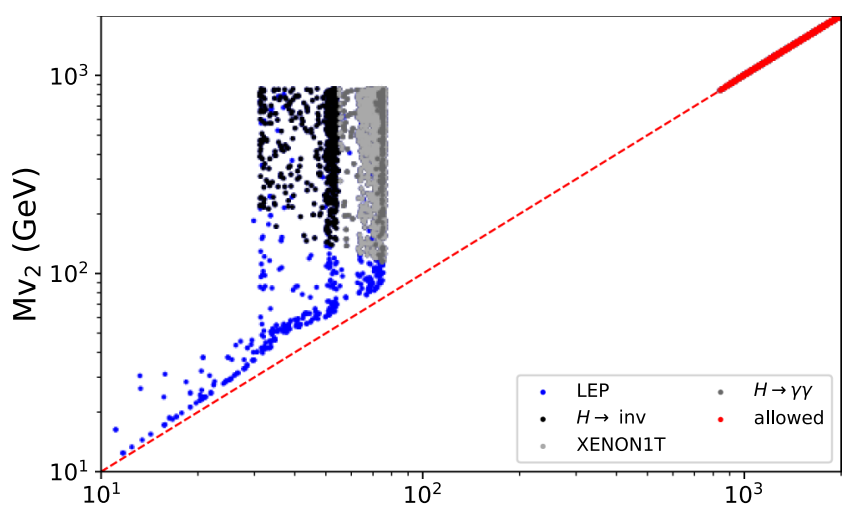

(b) $M v_{1}(\mathrm{GeV})$

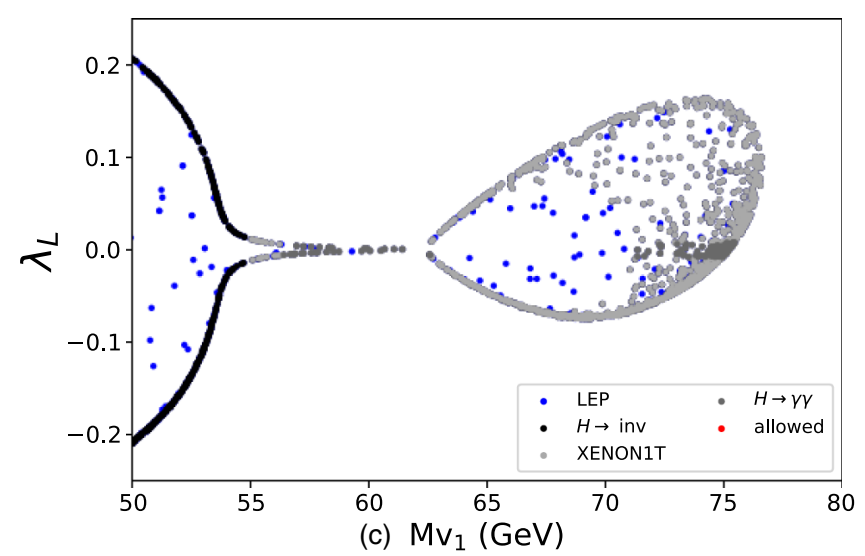

FIG. 7. 2-D projections of the parameter space in two different planes. In here all the points fit the Planck relic abundance, however only the red points survive all the restrictions. In (a) and (b) we show the full scan with masses between 10 and $2000 \mathrm{GeV}$, and a zoomed version of the low mass region is shown in (c).

projection of the 4-dimensional parameter space for the planes $\left(M_{V^{1}}, \lambda_{L}\right)$ and $\left(M_{V^{1}}, M_{V^{2}}\right)$, where we show all the points which can saturate the Planck limit but only the red points surviving all the restrictions mentioned above, and the other color points specifying which restriction excludes them.

There are two regions where the vector DM can satisfy the relic density constraints. The first one happens in the low DM mass region between $10 \lesssim M_{V^{1}} \lesssim 80 \mathrm{GeV}$, however this zone is complete excluded by the experimental constraints. As we showed previously in Sec. IVA, the production cross section for vectors present an enhancement due to their longitudinal polarization in comparison with the scalar case (i2HDM model), therefore the recast limits coming from LEP-II, the blue points, are more restrictive in our model than in the i2HDM, allowing just a narrow region close to the LEP energy threshold (1). Besides this, when $M_{V^{1}}<M_{H} / 2$ the restrictions coming from the invisible Higgs decay are extremely strong and only those cases where $\lambda_{L} \lesssim 10^{-2}$ can survive the restrictions coming from (19). The black points of Fig. 7 show the exclusion region by this constrain below $55 \mathrm{GeV}$ where the values of $\lambda_{L}$ take greater values. On the other hand, direct detection constraints are more sensitive in the low DM mass region, therefore only values for $\lambda_{L} \sim 0$ are compatible with this constraint. The light grey points are excluded by DD and only a small corridor between $55<M_{V 1}<$ $63 \mathrm{GeV}$ and $72<M_{V 1}<76 \mathrm{GeV}$ is allowed. Finally, the remaining points in the lower DM mass region are excluded by the restrictions from $H \rightarrow \gamma \gamma$. In this narrow region when $\lambda_{L}$ is very near to zero the mass difference between $V_{1}$ and $V^{ \pm}$can be high enough to increase the value of $\lambda_{2}$ and then increasing the diphoton rate beyond the allowed by the experimental data given by (18). Therefore, the low dark matter mass is completely excluded by the current experimental data.

The DM mass region which survive after all the experimental restrictions is located at the high DM mass zone, i.e., $M_{V^{1}} \gtrsim 840 \mathrm{GeV}$, as we can see from Fig. 7. This result contrasts with the one found in Refs. [29,30] where the dark vector can only explain partially the DM relic abundance, at least below the $\mathrm{TeV}$ scale. One of the most important features of this regions is the high level of degeneracy between the vector masses showed in the plane 


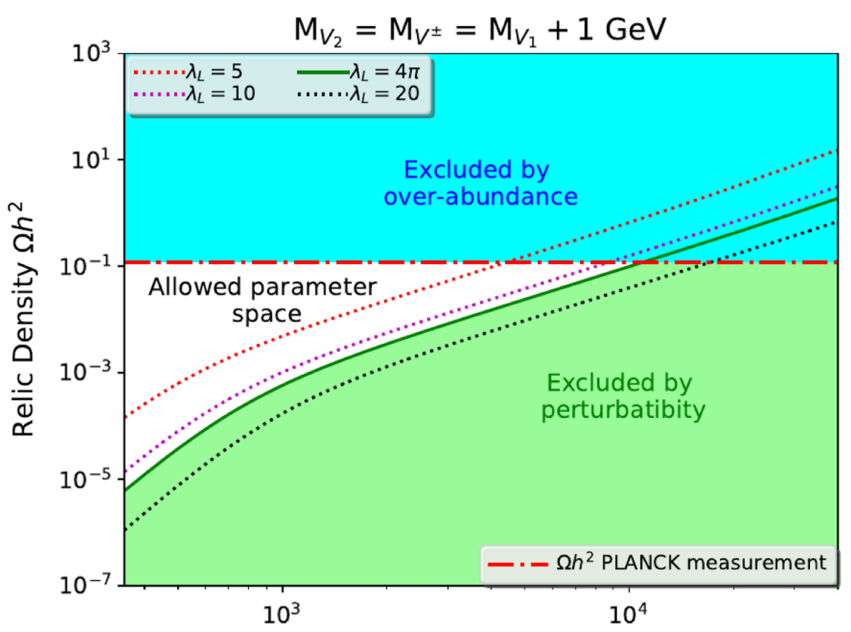

(a) $\mathrm{Mv}_{1}(\mathrm{GeV})$

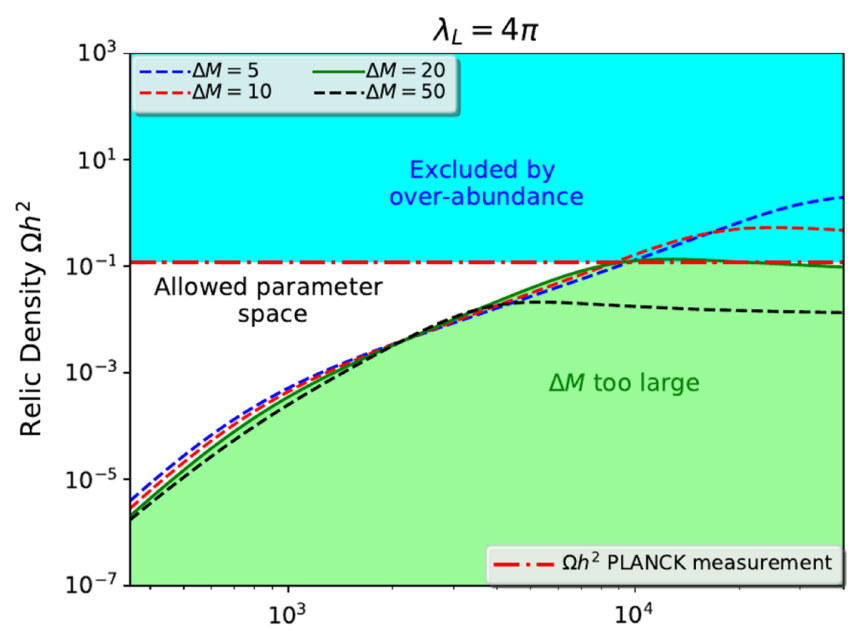

(b) $\mathrm{Mv}_{1}(\mathrm{GeV})$

FIG. 8. Closing of the parameter space at high values of $M_{V^{1}}$ and $\lambda_{L}$ in a quasidegenerate scenario.

$\left(M_{V^{1}}, M_{V^{2}}\right)$ of Fig. 7(b), where the mass splitting does not exceed $\sim 20 \mathrm{GeV}$.

Despite the fact that the direct detection experiment is less sensitive in the zone of high DM mass, the XENON1T constraints are still able to exclude parameter space for $\lambda_{L} \gtrsim 0.3$ in this zone. As the value of $M_{V^{1}}$ increases and DD loses sensibility, the allowed region becomes bigger, and higher $\lambda_{L}$ values are allowed. This is appreciated as the gray region for $M_{V^{1}} \gtrsim 840 \mathrm{GeV}$ in Fig. 7(a).

As $M_{V^{1}}$ increases in this scenario of high degeneracy, $\lambda_{L}$ can take more and more great values. However, when the DM mass is approaching much higher mass $(\sim 10 \mathrm{TeV}), \lambda_{L}$ starts to be outside the perturbativity constraints (12). Now, with this value of $\lambda_{L}$, the difference of masses among DM and the other vectors can only reach up to $20 \mathrm{GeV}$, after that point the quartic couplings become too large making the effective DM annihilation cross section fall below the experimental value of Planck. This completely closes the parameter space of the model as we can see from Fig. 8.

\section{B. Dark matter production at the LHC}

The DM double production associated with either monojet $j$, mono- $Z$ or a mono- $H$ are signals expected to be seen at the LHC in the context of dark matter searches. Due to the similarities in the topology of these processes between our model and the well-known inert-two-Higgsdoublet-model (i2HDM) [33,37], we compare the parton level distribution cross section and the missing transverse energy shape in mono-X $(j, Z, H)$ processes. ${ }^{4}$ The calculations were made with CALCHEP package, using NNPDF23_10_as_0130_qed (proton) as a parton

\footnotetext{
${ }^{4}$ Detailed analysis of DM production at LHC considering theses processes in the i2HDM, see [37], and a more fine analysis for monojet signature at the LHC, see [55].
}

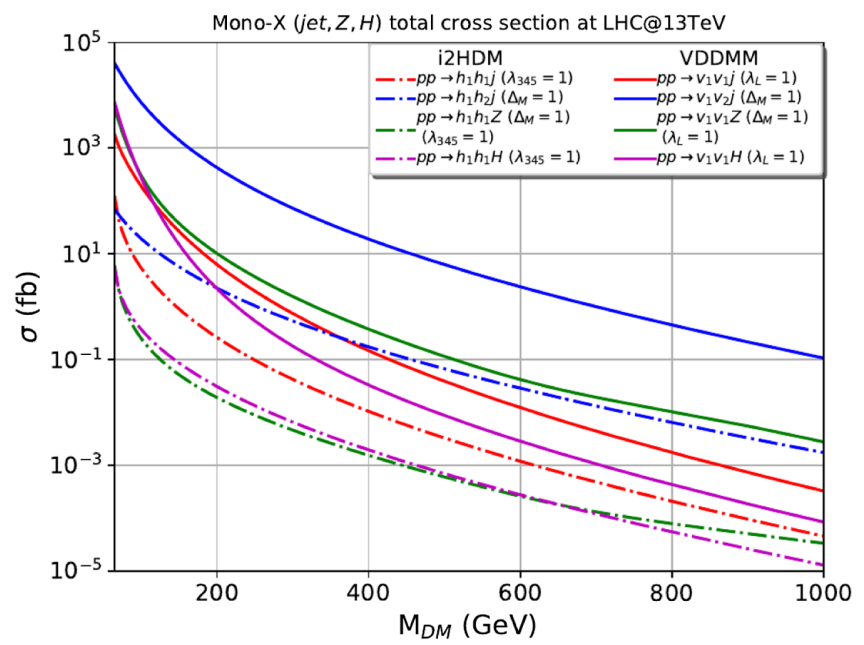

FIG. 9. Mono-X $(Z, j, H)$ cross section as a function of the DM mass. The dashed lines correspond to the scalar case (i2HDM) and the continuous one to the vectorial one (DVDM).

distribution function, and a generic transverse momentum cut of $100 \mathrm{GeV}$ on each of the SM particles.

In Fig. 9 we show the total cross section for the aforementioned processes as a function of the DM mass in both models for LHC@13 TeV. The continuous lines correspond to the case of vector DM (DVDM), whereas the dashed lines to the scalar case (i2HDM). All the processes consider the quasidegenerate mass scenario $\Delta M=1$ and $\lambda_{345}\left(\lambda_{L}\right)=1$.

Because the topology of the Feynman diagrams in both models are exactly the same in all the processes studied here, ${ }^{5}$ the differences lie mainly in the spin of the final

\footnotetext{
${ }^{5}$ The additional scalar states in the i2HDM are equivalent to the DVDM model, just do the replacement $V^{1,2} \rightarrow h_{1,2}$ and $V^{ \pm} \rightarrow h^{ \pm}$.
} 


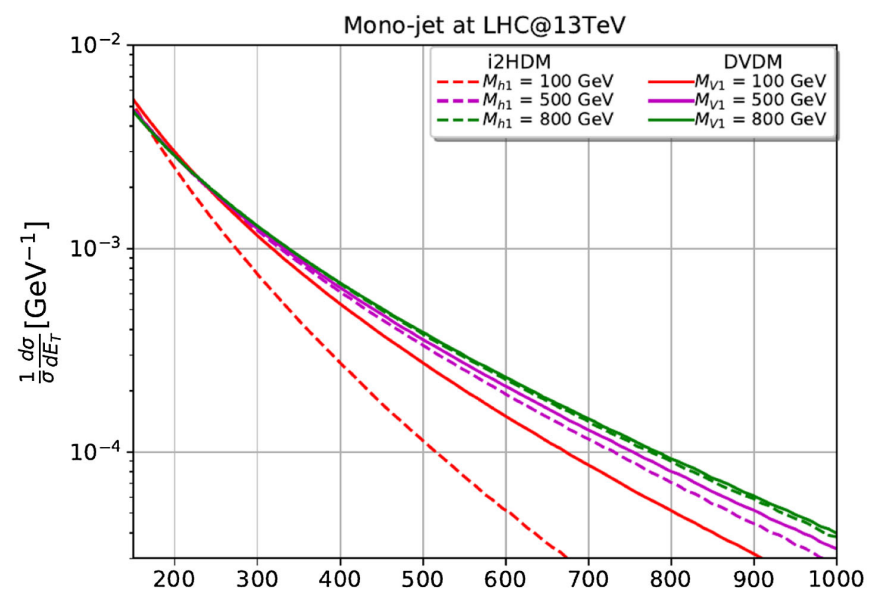

(a) $\mathrm{E}_{T}^{\text {miss }}[\mathrm{GeV}]$

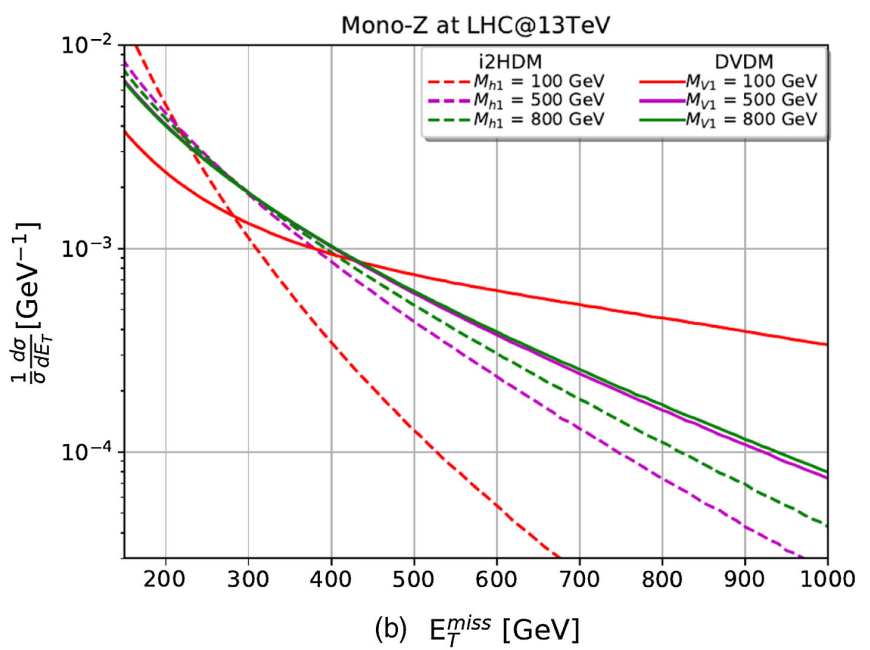

(b) $\mathrm{E}_{T}^{\text {miss }}[\mathrm{GeV}]$

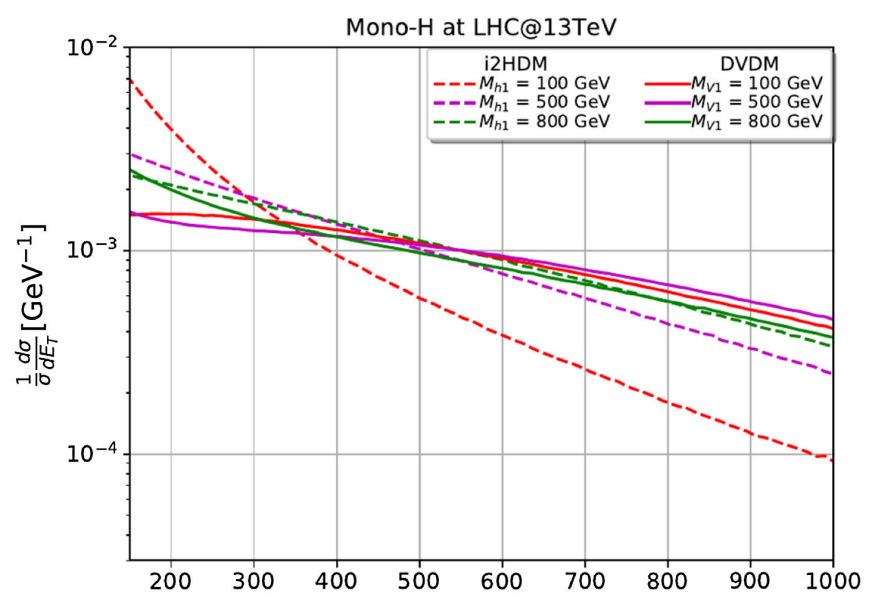

(c) $\mathrm{E}_{T}^{\text {miss }}[\mathrm{GeV}]$

FIG. 10. Normalized differential missing transverse energy cross section for the processes $p p \rightarrow X+E(X=j, Z, H)$ in i2HDM and DVDM. The dashed lines correspond to the predictions of the i2HDM and the continuous one in the DVDM. All the plots contain different DM mass: 100,500 and $800 \mathrm{GeV}$, in the quasidegenerate case, i.e., $\Delta M=1 \mathrm{GeV}, \lambda_{345}\left(\lambda_{L}\right)=0.1$, and at $\sqrt{s}=13 \mathrm{TeV} \mathrm{LHC}$ energies. A $p_{T, X} \geq 100 \mathrm{GeV}$ cut has been applied in all the plots.

states. The dependence of the cross section on the DM mass is similar in both cases. However the vector case is scaled up over the scalar one by, roughly, two orders of magnitude. This vector cross section enhancement is due to the fact that the longitudinal polarization of vectors scale as $\sim E / M_{V}$, implying that the production matrix element receive a significant enhancement in the region of phase space where the DM state is relativistic and either one or both particles are longitudinally polarized.

On the other hand, in Fig. 10 we show the normalized missing transverse energy distribution cross section of each one of the processes at parton level, considering the same mass splitting $\Delta M=1 \mathrm{GeV}$ and $\lambda_{L}=0.1$. In each channel, the distributions for the vector case are always flattened with respect to the scalar ones. This behavior is in agreement with the results presented in [6]. Furthermore, the differences in the shapes are more notorious in the cases in which the new state masses are lower. Considering that monojet signals have the higher cross sections, we complement the analysis with the invariant mass distribution of the DM pairs. In Fig. 11 we present $M_{\text {inv }}(D M, D M)$ distributions for the scalar and vector cases in the monojet case, again normalized to unity for $\sqrt{s}=13 \mathrm{TeV}$ LHC energies. From Fig. 11, one can see that the $M_{\text {inv }}(D M, D M)$ distributions are better separated for higher masses of scalars and vectors. The scalar distributions are closer to the point $M_{\text {inv }}(D M, D M)=2 M_{\mathrm{DM}}$, whereas the vectorial ones distributions are broader.

\section{PERTURBATIVE UNITARITY}

Having shown that our model can provide a viable dark matter candidate, we want to discuss the validity range of our effective approach. The main theoretical challenge 


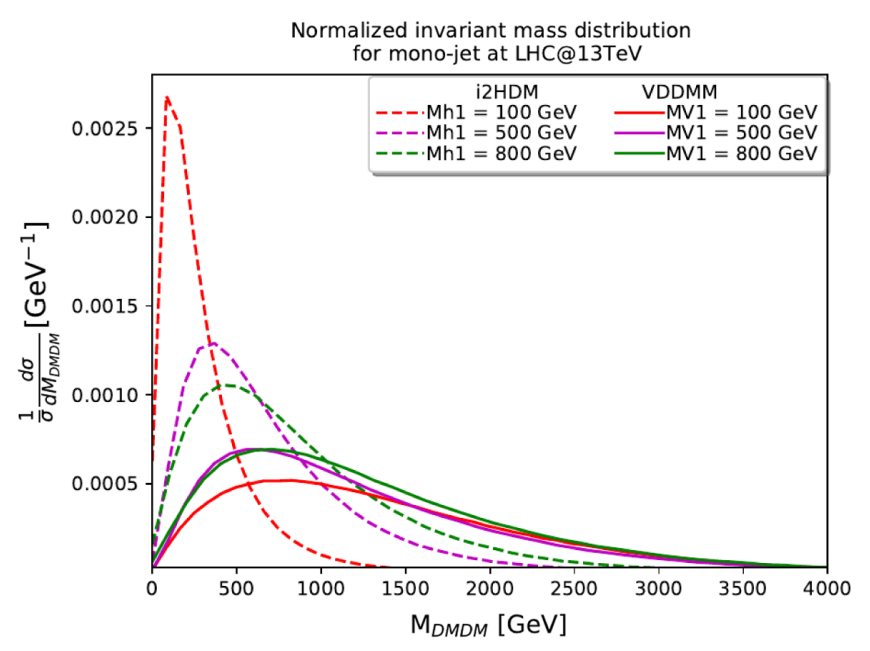

FIG. 11. Invariant mass of DM pair distributions normalized to unity for monojet in both i2HDM (dashed) and DVDM (continuous) at $13 \mathrm{TeV}$ LHC energy. All the results are considering $\lambda_{345}\left(\lambda_{L}\right)=0.1$, in the quasidegenerate case, i.e., $\Delta M=1 \mathrm{GeV}$.

faced by our construction is the eventual violation of perturbative unitarity introduced by the new massive vector states. To this aim, we study the amplitudes, in the high energy regime, of representative and potentially problematic processes like $h V^{1} \rightarrow h V^{1}$ and $Z V^{ \pm} \rightarrow Z V^{ \pm}$.

In Fig. 12(a) is shown the maximum energy scale at which the process $h V^{1} \rightarrow h V^{1}$ is valid until perturbative unitarity starts to be violated. As $\lambda_{L}$ gets smaller the bigger is the scale energy before the breaking of perturbative unitarity. Additionally, the bound on the energy gets relax as $M_{V^{1}}$ raises too. For values of $M_{V^{1}}$ below $100 \mathrm{GeV}$ the scale of unitarity violation is mostly constant and of the order of a few $\mathrm{TeVs}$, whereas for higher masses the dependence on $\lambda_{L}$ starts to grow, making our model consistent at scales as high as $10 \mathrm{TeV}$ for small values of $\lambda_{L}$. Therefore, from the point of view of unitarity, our construction is perfectly safe for masses of the DM candidate above $200 \mathrm{GeV}$ specially when $\lambda_{L}$ to is small. We want to remark that the phenomenologically interesting region of the space parameter, where our DM candidate saturates the relic density, belongs to the unitarity safe zone.

In Fig. 12(b) is shown the maximum energy scale in the plane $\left(\lambda_{L}, M_{V^{ \pm}}\right)$at which the process $Z_{L} V_{L}^{ \pm} \rightarrow Z_{L} V_{L}^{ \pm}$is valid until perturbative unitarity is violated. At masses near $100 \mathrm{GeV}$ and $\lambda_{L}$ close to zero, the maximum energy values allowed by perturbative unitarity rises easily above $5 \mathrm{TeV}$. On the other hand, for values of $M_{V^{ \pm}}$near $1 \mathrm{TeV}$, the scale of unitarity violation is of the order of $3 \mathrm{TeV}$.

These results are consistent with unitarity analysis of some vector dark matter models $[19,28]$ and it suggests that our effective model must meet an ultraviolet completion at a scale between 3 and $10 \mathrm{TeV}$. For instance, one of the simplest ways to restore unitarity is to embed our model into a larger gauge symmetry spontaneously broken by a new scalar sector [62]. In this sense, our model can be considered as a simplified model [12], retaining just the lightest states predicted in this scenario, and pushing the required new states at scales above the vectorial ones.

\section{CONCLUSIONS}

Unlike most of extensions to the Standard Model which consider new massive vector fields as singlets or triplets under $S U(2)_{L}$ gauge group, in this work we have explored a different possibility. The new vector degrees of freedom enter into the SM in the fundamental representation of $S U(2)_{L}$, with hypercharge $Y=1 / 2$. Unlike the vector triplet case, our model accepts a potential composed of many terms coupling the new vector to the Higgs doublet with independent coupling constants. This feature makes the model more similar to the i2HDM than to models with vector triplets. Additionally, due to the quantum numbers assigned to the new vector, it is impossible to couple it to standard fermions through renormalizable operator. The model acquires a $Z_{2}$ symmetry in the limit in which the

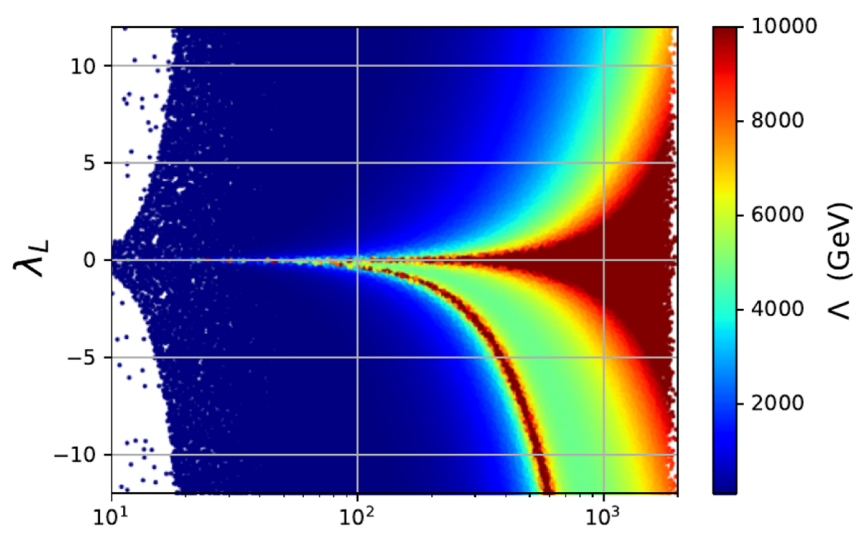

(a) $M v_{1}(\mathrm{GeV})$

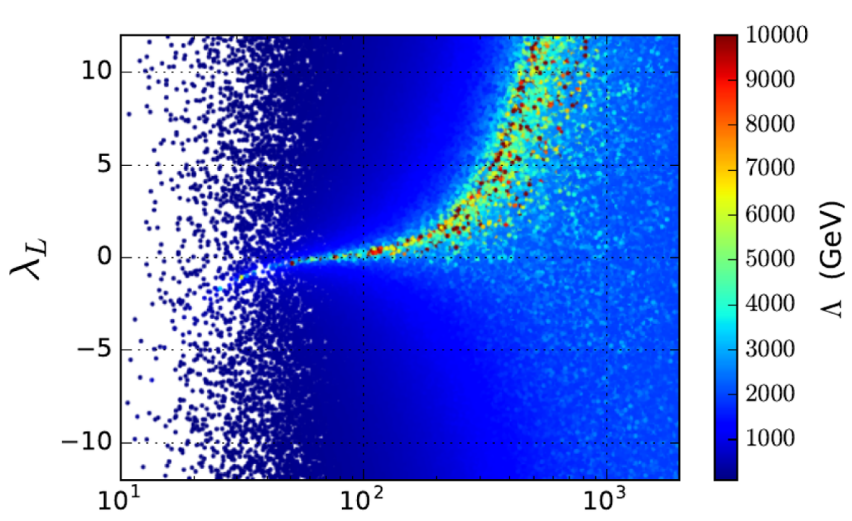

(b) $\mathrm{Mv}_{p}(\mathrm{GeV})$

FIG. 12. (a) Maximum energy-scale $\Lambda$ until the process $h V^{1} \rightarrow h V^{1}$ starts to violate perturbative unitarity. (b) Maximum energy-scale $\Lambda$ until the process $Z V^{ \pm} \rightarrow Z V^{ \pm}$starts to violate perturbative unitarity. 
only nonstandard dimension three operator is eliminated. This choice is natural in the sense of t'Hooft and allows the neutral vector $V^{1}$ to be a good dark matter candidate.

We have performed a detailed analysis constraining the model through LEP and LHC data, DM relic density, direct and indirect DM detection. We found that the main experimental constraints are imposed by recent measurement of $H \rightarrow \gamma \gamma$ (mainly when the $V^{1}$ is light) and data on direct search of DM obtained by XENON1T.

After imposing all the experimental constraints, we found that for a range of masses between $840 \leq M_{V^{1}} \leq$ $10^{4} \mathrm{GeV}$ in the highly degenerate case where $\Delta M<$ $20 \mathrm{GeV}$ the lightest neutral component of the doublet can reach the relic density measurements (23), surviving all the experimental constraints. This contrasts with other electroweak vector multiplet models, where the saturation value for DM is above the TeV scale (see, e.g., [28,31]), or other models where the dark vector component never reach the DM budget (see, e.g., [29,30]). Furthermore, if we relax the lower Planck limit (23) allowing additional sources of dark matter, there is an important sector of the parameter space for $M_{V^{1}} \gtrsim 100 \mathrm{GeV}$ that it is still not possible to rule out with the current experiments.

At this point, we want to dedicate some sentences to compare our construction to the recently proposed minimal vector dark matter model (MVDM) [28]. In both models, the dark matter candidate is a component of a vector field transforming a nontrivial representation of $S U(2)_{L}$ : the adjoint representation in the case of MVDM and the fundamental one in our case. The difference in the field representation makes a significant separation between the two models. The most evident one is related to the number of new vector states (three for the MVDM and four in our case). But more important is what happens with the potential in the Higgs-massive-vector sector. In the MVDM this sector is extremely simple, contributing with only one term to the Lagrangian and only one of the two free parameters of the model. In our case, the scalar-vector potential is richer with three free parameters. This is, in part, the origin of the different ultraviolet behavior reflected in the scale of unitarity violation which is systematically larger in the MVDM. In fact, the structure of the potential of our model makes it more closely related to the i2HDM than to the MVDM making harder to differentiate our model from the former than from the latter.

In view of the similarities between i2HDM with our model, we compared the parton level cross section and the normalized missing energy differential cross section for mono- $X(\mathrm{jet}, Z, H)$. Mono-X cross sections get enhanced in the vectorial case due to their growing energy behavior of their final state longitudinal polarization. The shapes of the distribution of missing energy results to be flatter in the vectorial cases. This feature may help to distinguish between our model and the i2HDM.
Finally, as a complement to this work, we have shown some results of perturbative unitarity bounds on some scattering amplitudes involving the new states. Our analysis suggests that our effective approach needs an ultraviolet completion at a scale of the order of 3 to $10 \mathrm{TeV}$.

\section{ACKNOWLEDGMENTS}

We would like to thank Marcela González for contributions at early stages of this work, and also to Alexander Belyaev, Eduardo Pontón and Sebastián Norero for valuable discussions. B. D. was supported partially by Conicyt Becas Chile and DGIIP-UTFSM. A. Z. was supported in part by Conicyt (Chile) Grants No. PIA/ACT-1406 and No. PIA/Basal FB0821, and by Fondecyt (Chile) Grant No. 1160423. F. R. was supported by Conicyt Becas Chile Postdoctorado Grant No. 74180065. A. Z. is very thankful to the developers of MAXIMA [63] and the package Dirac2 [64]. These softwares were used in parts of this work.

\section{APPENDIX: MORE ON PARTIAL WAVES AMPLITUDES}

In this Appendix we complement the results of the analysis presented in Sec. VI with more details. Additionally, we make some comments about perturbative unitarity on selfinteraction amplitudes $\left(V V \rightarrow V V\right.$, for $V=V^{1}, V^{2}$ or $\left.V^{ \pm}\right)$.

In Fig. 13 is shown the value of the scale of perturbative unitarity violation $(\Lambda)$ for the process $h V^{1} \rightarrow h V^{1}$ in the planes $\left(M_{V^{1}}, M_{V^{2}}\right),\left(M_{V^{1}}, M_{V^{ \pm}}\right)$, and $\left(M_{V^{2}}, M_{V^{ \pm}}\right)$, respectively. In Table III we resume the zero partial wave for the three possible elastic scattering of this type. In concordance with the information given by Fig. 12(a), for lower masses $(\lesssim 200 \mathrm{GeV})$, the values of $\Lambda$ are located around the TeV energy scale for most of the masses combinations allowed by experimental constrains. For higher masses, $\Lambda$ stars to grow for most of possible combination of masses, and there is a slightly raising in the energy as the degeneracy among the three states becomes similar.

On the other hand, in Fig. 14 we present different plots showing the values of $\Lambda$ for the process $Z V^{ \pm} \rightarrow Z V^{ \pm}$. In this case, the degeneracy of the states do not show any raising in the maximum allowed energy value. According to what is shown in Fig. 12(b), as the masses get near the $\mathrm{TeV}$ scale, $\Lambda$ gets a constant value near $4 \mathrm{TeV}$, making this process more stringent for masses above $\sim 500 \mathrm{GeV}$ than the previous one with the Higgs involved.

Finally, we make some comments about the $V_{L}+V_{L} \rightarrow$ $V_{L}+V_{L}$ amplitudes, for $V=V^{1}, V^{2}$, and $V^{ \pm}$. These processes may introduce strong constraints on the energy scale at which perturbative unitarity breaks down. For example, let us first consider the process $V_{L}^{1}+V_{L}^{1} \rightarrow V_{L}^{1}+V_{L}^{1}$. Its zero partial wave is 


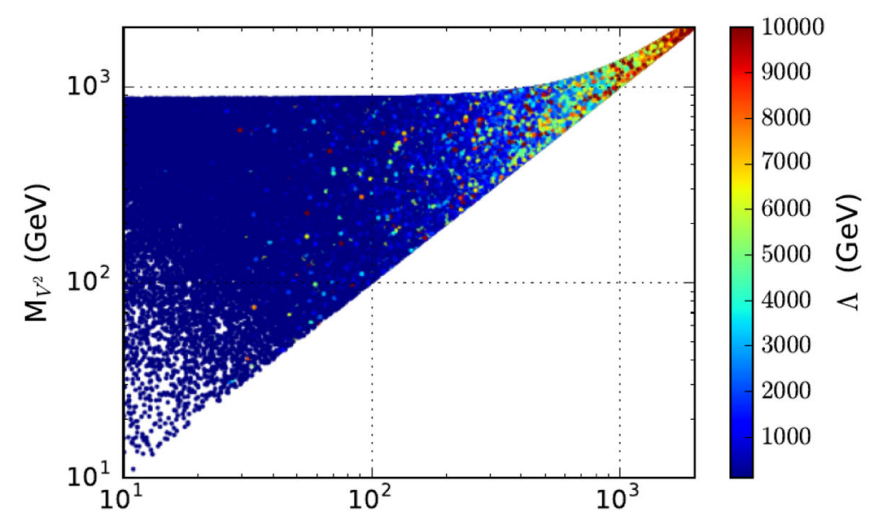

(a) $M_{V^{1}}(\mathrm{GeV})$

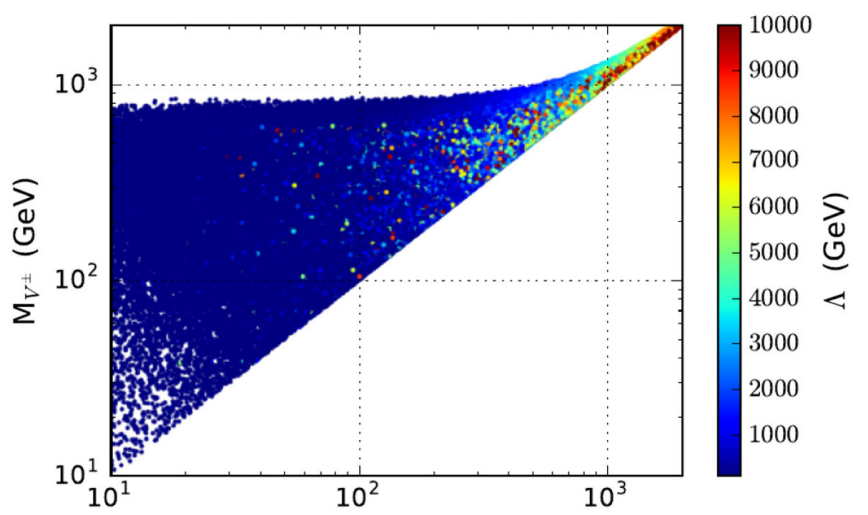

(b) $M_{V^{1}}(\mathrm{GeV})$

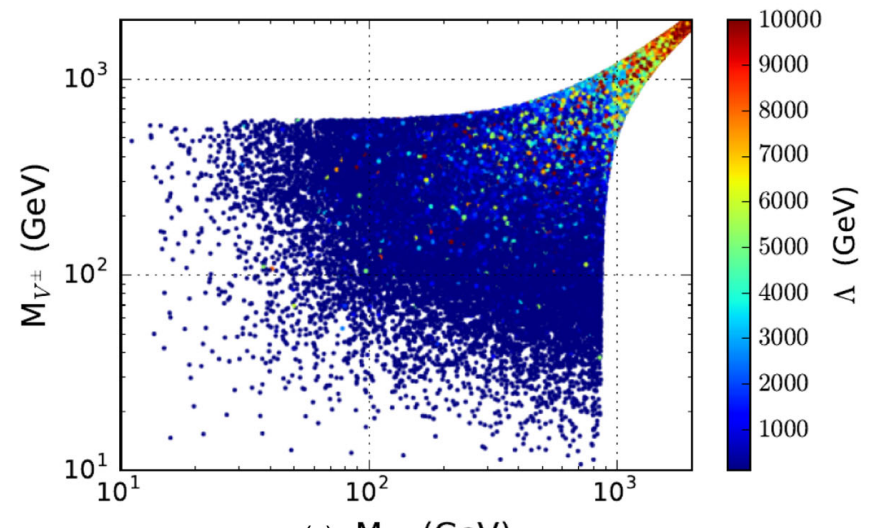

(c) $M_{V^{2}}(\mathrm{GeV})$

FIG. 13. Maximum allowed energy $\Lambda$ by perturbative unitarity bounds on $h V^{1} \rightarrow h V^{1}$ amplitude. The plots are projected in the planes $\left(M_{V^{1}}, M_{V^{2}}\right)(\mathrm{a}),\left(M_{V^{1}}, M_{V^{ \pm}}\right)(\mathrm{b})$, and $\left(M_{V^{2}}, M_{V^{ \pm}}\right)(\mathrm{c})$.

$a_{0}(s)=\frac{g^{2}\left(\alpha_{2}+\alpha_{3}\right)\left(36 M_{V^{1}}^{4}-24 M_{V^{1}}^{2} s+5 s^{2}\right)+18 \lambda_{L}^{2} M_{H}^{2} M_{W}^{2}}{96 \pi g^{2} M_{V^{1}}^{4}}$,

where $\alpha_{2}$ and $\alpha_{3}$ are the self-couplings among the new states [see (2)]. The strong growing energy behavior of the partial wave $\left(a_{0} \sim E^{4}\right)$ makes that perturbative unitarity breaks

TABLE III. Partial waves for $h V \rightarrow h V$ elastic tree level scatterings processes. Each of the three processes contain a contact diagram.

\begin{tabular}{lccc}
\hline \hline Process & s-cha & t-cha & Partial wave $\left(a_{0}\right)$ \\
\hline$h V^{1} \rightarrow h V^{1}$ & $V^{1}$ & $H, V^{1}$ & $-\frac{\lambda_{L} s}{64 \pi M_{V^{1}}^{2}}\left(1+2 \frac{\lambda_{L}}{g^{2}} \frac{M_{W}^{2}}{M_{V^{1}}^{2}}\right)$ \\
$h V^{2} \rightarrow h V^{2}$ & $V^{2}$ & $H, V^{2}$ & $-\frac{\lambda_{R} s}{64 \pi M_{V^{2}}^{2}}\left(1+2 \frac{\lambda_{R}}{g^{2}} \frac{M_{W}^{2}}{M_{V^{2}}^{2}}\right)$ \\
$h V^{ \pm} \rightarrow h V^{ \pm}$ & $V^{ \pm}$ & $H, V^{ \pm}$ & $-\frac{\lambda_{2} s}{64 \pi M_{V^{ \pm}}^{2}}\left(1+2 \frac{\lambda_{2}}{g^{2}} \frac{M_{W}^{2}}{M_{V^{ \pm}}^{2}}\right)$ \\
\hline \hline
\end{tabular}

down at very low energies for typical masses of a few hundred $\mathrm{GeV}$. For example, for $M_{V^{1}}=100 \mathrm{GeV}, \lambda_{L}=1$ and $\alpha_{1}=\alpha_{2}=1$, the breaking of perturbative unitarity is reached at energy scales less than $250 \mathrm{GeV}$. Interestingly, the growing energy behavior disappears when $\alpha_{1}=-\alpha_{2}$. However, under this last condition, the amplitude $V_{L}^{+}+$ $V_{L}^{-} \rightarrow V_{L}^{+}+V_{L}^{-}$still grows with the energy as $s^{2} \sim E^{4}$ :

$$
a_{0}(s)=\frac{g^{4}\left(9\left(1-2 c_{w}^{2}\right)^{2} M_{W}^{2} s-4 c_{w}^{2} s^{2}\right)+48 c_{w}^{4} M_{W}^{2} \lambda_{2} s}{1536 \pi g^{2} c_{w}^{4} M_{V^{ \pm}}^{4}},
$$

where $\lambda_{2}$ is a function of $\lambda_{L}, M_{V^{1}}$, and $M_{V^{ \pm}}$[see Eq. (9)], and the lost of perturbative unitarity starts to be around $3 \mathrm{TeV}$. Therefore, it seems impossible to get rid of the growing energy behavior with an arbitrarily choose of the free parameters. As we have pointed out in Sec. VI, a possible solution to this problem is to establish the model from a gauge theory in order to generate a gauge cancellation among the $s$ - and $t$ - channels and the contact graph [65]. 


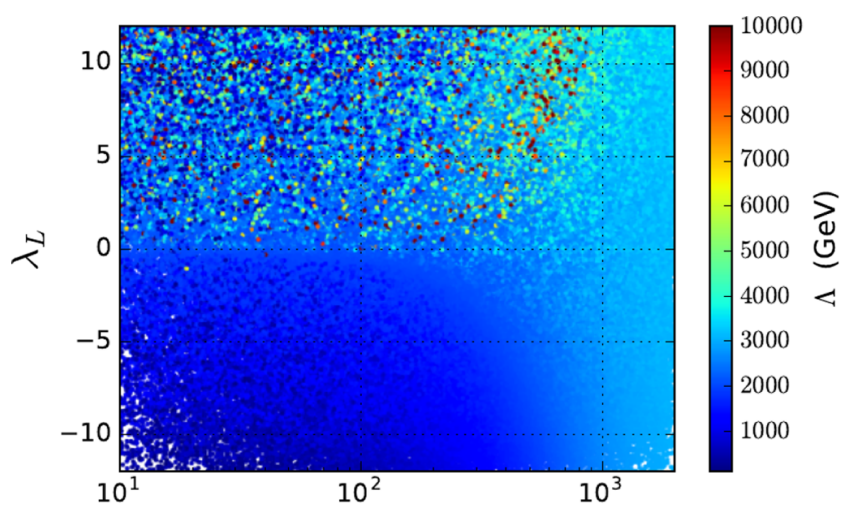

(a) $\mathrm{Mv}_{1}(\mathrm{GeV})$

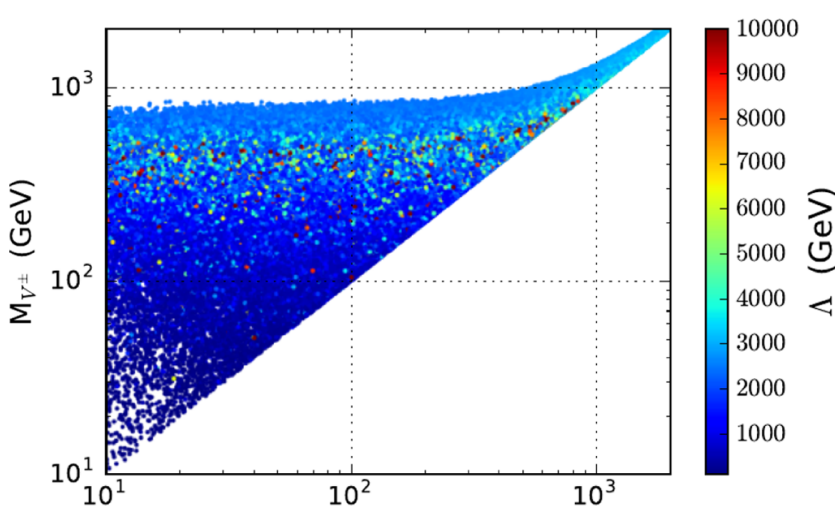

(c) $M_{V^{1}}(\mathrm{GeV})$

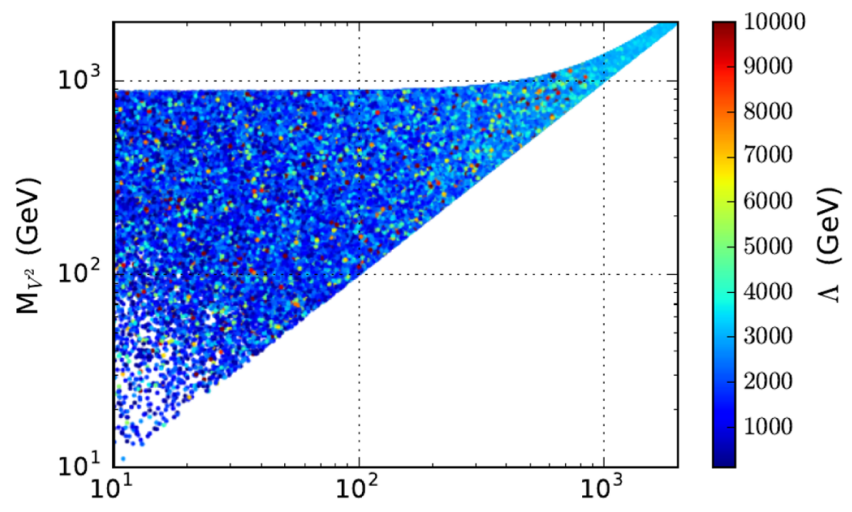

(b) $M_{V^{1}}(\mathrm{GeV})$

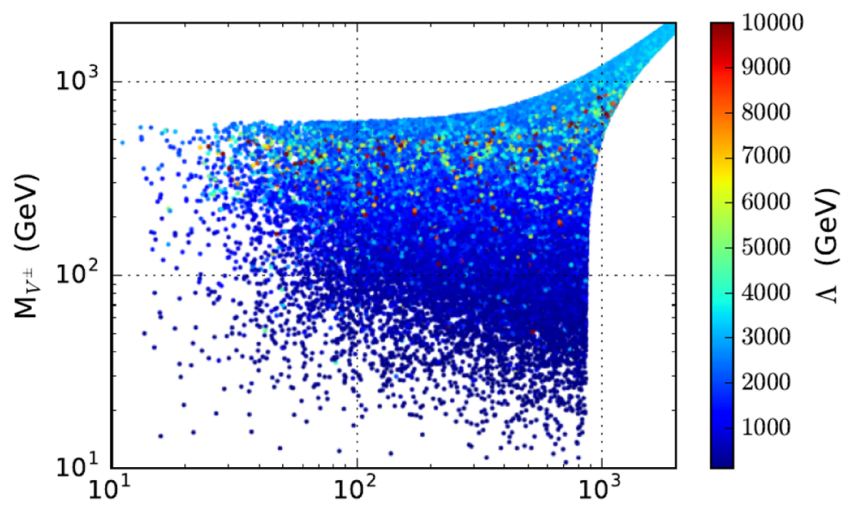

(d) $M_{V^{2}}(\mathrm{GeV})$

FIG. 14. Maximum allowed energy $\Lambda$ from perturbative unitarity bounds on the processes $Z V^{ \pm} \rightarrow Z V^{ \pm}$in the planes (a) $\left(M_{V^{1}}, M_{V^{2}}\right)$, (b) $\left(M_{V^{1}}, M_{V^{ \pm}}\right)$, and (c) $\left(M_{V^{2}}, M_{V^{ \pm}}\right)$.

[1] M. Beltran, D. Hooper, E. W. Kolb, Z. A. C. Krusberg, and T. M. P. Tait, Maverick dark matter at colliders, J. High Energy Phys. 09 (2010) 037.

[2] Y. Bai, P. J. Fox, and R. Harnik, The Tevatron at the Frontier of dark matter direct detection, J. High Energy Phys. 12 (2010) 048.

[3] A. L. Fitzpatrick, W. Haxton, E. Katz, N. Lubbers, and Y. $\mathrm{Xu}$, The effective field theory of dark matter direct detection, J. Cosmol. Astropart. Phys. 02 (2013) 004.

[4] G. Busoni, A. De Simone, E. Morgante, and A. Riotto, On the validity of the effective field theory for dark matter searches at the LHC, Phys. Lett. B 728, 412 (2014).

[5] G. Busoni, A. De Simone, J. Gramling, E. Morgante, and A. Riotto, On the validity of the effective field theory for dark matter searches at the LHC, Part II: Complete analysis for the $s$-channel, J. Cosmol. Astropart. Phys. 06 (2014) 060.

[6] A. Belyaev, L. Panizzi, A. Pukhov, and M. Thomas, Dark matter characterization at the LHC in the effective field theory approach, J. High Energy Phys. 04 (2017) 110.
[7] P. J. Fox, R. Harnik, J. Kopp, and Y. Tsai, Missing energy signatures of dark matter at the LHC, Phys. Rev. D 85, 056011 (2012).

[8] A. Rajaraman, W. Shepherd, T. M. P. Tait, and A. M. Wijangco, Lhc bounds on interactions of dark matter, Phys. Rev. D 84, 095013 (2011).

[9] M. R. Buckley, D. Feld, and D. Goncalves, Scalar simplified models for dark matter, Phys. Rev. D 91, 015017 (2015).

[10] O. Buchmueller, M. J. Dolan, and C. McCabe, Beyond effective field theory for dark matter searches at the LHC, J. High Energy Phys. 01 (2014) 025.

[11] O. Buchmueller, M. J. Dolan, S. A. Malik, and C. McCabe, Characterising dark matter searches at colliders and direct detection experiments: Vector mediators, J. High Energy Phys. 01 (2015) 037.

[12] J. Abdallah et al., Simplified models for dark matter searches at the LHC, Phys. Dark Universe 9-10, 8 (2015).

[13] J. Abdallah et al., Simplified models for dark matter and missing energy searches at the LHC, arXiv:1409.2893. 
[14] M. Cirelli, N. Fornengo, and A. Strumia, Minimal dark matter, Nucl. Phys. B753, 178 (2006).

[15] T. Hambye, Hidden vector dark matter, J. High Energy Phys. 01 (2009) 028.

[16] J. L. Diaz-Cruz and E. Ma, Neutral SU(2) gauge extension of the Standard Model and a vector-boson dark-matter candidate, Phys. Lett. B 695, 264 (2011).

[17] S. Kanemura, S. Matsumoto, T. Nabeshima, and N. Okada, Can WIMP dark matter overcome the nightmare scenario, Phys. Rev. D 82, 055026 (2010).

[18] A. Djouadi, O. Lebedev, Y. Mambrini, and J. Quevillon, Implications of LHC searches for Higgs-portal dark matter, Phys. Lett. B 709, 65 (2012).

[19] O. Lebedev, H. M. Lee, and Y. Mambrini, Vector Higgsportal dark matter and the invisible Higgs, Phys. Lett. B 707, 570 (2012).

[20] Y. Farzan and A. R. Akbarieh, VDM: A model for vector dark matter, J. Cosmol. Astropart. Phys. 10 (2012) 026.

[21] S. Baek, P. Ko, W.-I. Park, and E. Senaha, Higgs portal vector dark matter: Revisited, J. High Energy Phys. 05 (2013) 036.

[22] J.-H. Yu, Vector fermion-portal dark matter: Direct detection and galactic center gamma-ray excess, Phys. Rev. D 90, 095010 (2014).

[23] C. Gross, O. Lebedev, and Y. Mambrini, Non-Abelian gauge fields as dark matter, J. High Energy Phys. 08 (2015) 158.

[24] S. Di Chiara and K. Tuominen, A minimal model for SU(N) vector dark matter, J. High Energy Phys. 11 (2015) 188.

[25] G. Servant and T. M. P. Tait, Is the lightest Kaluza-Klein particle a viable dark matter candidate, Nucl. Phys. B650, 391 (2003).

[26] A. Birkedal, A. Noble, M. Perelstein, and A. Spray, Little Higgs dark matter, Phys. Rev. D 74, 035002 (2006).

[27] T. Abe, M. Kakizaki, S. Matsumoto, and O. Seto, Vector WIMP miracle, Phys. Lett. B 713, 211 (2012).

[28] A. Belyaev, G. Cacciapaglia, J. Mckay, D. Marin, and A. R. Zerwekh, Minimal spin-one isotriplet dark matter, arXiv:1808.10464.

[29] J. K. Mizukoshi, C. A. de S. Pires, F. S. Queiroz, and P. S. Rodrigues da Silva, WIMPs in a 3-3-1 model with heavy sterile neutrinos, Phys. Rev. D 83, 065024 (2011).

[30] P. V. Dong, D. T. Huong, F. S. Queiroz, J. W. F. Valle, and C. A. Vaquera-Araujo, The dark side of flipped trinification, J. High Energy Phys. 04 (2018) 143.

[31] N. Maru, N. Okada, and S. Okada, $S U(2)_{L}$ doublet vector dark matter from gauge-Higgs unification, Phys. Rev. D 98, 075021 (2018).

[32] N. G. Deshpande and E. Ma, Pattern of symmetry breaking with two higgs doublets, Phys. Rev. D 18, 2574 (1978).

[33] R. Barbieri, L. J. Hall, and V.S. Rychkov, Improved naturalness with a heavy Higgs: An alternative road to LHC physics, Phys. Rev. D 74, 015007 (2006).

[34] A. Goudelis, B. Herrmann, and O. Stal, Dark matter in the inert doublet model after the discovery of a Higgs-like boson at the LHC, J. High Energy Phys. 09 (2013) 106.

[35] A. Arhrib, Y.-L. S. Tsai, Q. Yuan, and T.-C. Yuan, An updated analysis of inert Higgs doublet model in light of the recent results from LUX, PLANCK, AMS-02 and LHC, J. Cosmol. Astropart. Phys. 06 (2014) 030.
[36] A. Ilnicka, M. Krawczyk, and T. Robens, Inert Doublet Model in light of LHC Run I and astrophysical data, Phys. Rev. D 93, 055026 (2016).

[37] A. Belyaev, G. Cacciapaglia, I. P. Ivanov, F. Rojas-Abatte, and M. Thomas, Anatomy of the inert two Higgs doublet model in the light of the LHC and non-LHC dark matter searches, Phys. Rev. D 97, 035011 (2018).

[38] J. L. Hewett, T. G. Rizzo, S. Pakvasa, H. E. Haber, and A. Pomarol, Vector leptoquark production at hadron colliders, in Workshop on Physics at Current Accelerators and the Supercollider Argonne, Illinois, 1993 (1993), pp. 0539-546.

[39] L. Lopez Honorez, E. Nezri, J. F. Oliver, and M. H. G. Tytgat, The inert doublet model: An archetype for dark matter, J. Cosmol. Astropart. Phys. 02 (2007) 028.

[40] S. Tulin and H.-B. Yu, Dark matter self-interactions and small scale structure, Phys. Rep. 730, 1 (2018).

[41] A. Semenov, LanHEP - a package for automatic generation of Feynman rules from the Lagrangian. Updated version 3.1, arXiv:1005.1909.

[42] A. Belyaev, N. D. Christensen, and A. Pukhov, CalcHEP 3.4 for collider physics within and beyond the Standard Model, Comput. Phys. Commun. 184, 1729 (2013).

[43] G. Belanger, F. Boudjema, A. Pukhov, and A. Semenov, micrOMEGAs_3: A program for calculating dark matter observables, Comput. Phys. Commun. 185, 960 (2014).

[44] G. Belanger, F. Boudjema, A. Pukhov, and A. Semenov, MicrOMEGAs 2.0: A program to calculate the relic density of dark matter in a generic model, Comput. Phys. Commun. 176, 367 (2007).

[45] G. Belanger, F. Boudjema, P. Brun, A. Pukhov, S. RosierLees, P. Salati, and A. Semenov, Indirect search for dark matter with micrOMEGAs2.4, Comput. Phys. Commun. 182, 842 (2011).

[46] Q.-H. Cao, E. Ma, and G. Rajasekaran, Observing the dark scalar doublet and its impact on the Standard-Model Higgs boson at colliders, Phys. Rev. D 76, 095011 (2007).

[47] M. Gustafsson, E. Lundstrom, L. Bergstrom, and J. Edsjo, Significant gamma lines from inert Higgs dark matter, Phys. Rev. Lett. 99, 041301 (2007).

[48] E. Lundström, M. Gustafsson, and J. Edsjö, Inert doublet model and LEP II limits, Phys. Rev. D 79, 035013 (2009).

[49] A. Pierce and J. Thaler, Natural dark matter from an unnatural Higgs boson and new colored particles at the TeV scale, J. High Energy Phys. 08 (2007) 026.

[50] P. Abreu et al. (DELPHI Collaboration), Search for neutralino pair production at $\mathrm{s} * *(1 / 2)=189-\mathrm{GeV}$, Eur. Phys. J. C 19, 201 (2001).

[51] G. Abbiendi et al. (OPAL Collaboration), Search for chargino and neutralino production at $\mathrm{S} * *(1 / 2)=$ 189-GeV at LEP, Eur. Phys. J. C 14, 187 (2000); Erratum, Eur. Phys. J. C16, 707(E) (2000).

[52] M. Aaboud et al. (ATLAS Collaboration), Measurements of Higgs boson properties in the diphoton decay channel with $36 \mathrm{fb}^{-1}$ of $p p$ collision data at $\sqrt{s}=13 \mathrm{TeV}$ with the ATLAS detector, Phys. Rev. D 98, 052005 (2018).

[53] G. Aad et al. (ATLAS Collaboration), Search for invisible decays of a Higgs boson using vector-boson fusion in $p p$ collisions at $\sqrt{s}=8 \mathrm{TeV}$ with the ATLAS detector, J. High Energy Phys. 01 (2016) 172. 
[54] V. Khachatryan et al. (CMS Collaboration), Searches for invisible decays of the Higgs boson in pp collisions at $\sqrt{s}=7,8$, and 13 TeV, J. High Energy Phys. 02 (2017) 135.

[55] A. Belyaev, T. R. Fernandez Perez Tomei, P. G. Mercadante, C. S. Moon, S. Moretti, S. F. Novaes, L. Panizzi, F. Rojas, and M. Thomas, Advancing LHC probes of dark matter from the inert 2-Higgs doublet model with the mono-jet signal, Phys. Rev. D 99, 015011 (2019).

[56] P. Ade et al. (Planck Collaboration), Planck 2015 results. XIII. Cosmological parameters, Astron. Astrophys. 594, A13 (2016).

[57] E. Aprile et al. (XENON Collaboration), First dark matter search results from the XENON1T experiment, Phys. Rev. Lett. 119, 181301 (2017).

[58] H. Abdallah et al. (H.E.S.S. Collaboration), Search for Dark Matter Annihilations towards the Inner Galactic Halo from 10 Years of Observations with H.E.S.S., Phys. Rev. Lett. 117, 111301 (2016).

[59] M. Ackermann et al. (Fermi-LAT Collaboration), Searching for Dark Matter Annihilation from Milky Way Dwarf
Spheroidal Galaxies with Six Years of Fermi Large Area Telescope Data, Phys. Rev. Lett. 115, 231301 (2015).

[60] G. Giesen, M. Boudaud, Y. Génolini, V. Poulin, M. Cirelli, P. Salati, and P. D. Serpico, AMS-02 antiprotons, at last! Secondary astrophysical component and immediate implications for Dark Matter, J. Cosmol. Astropart. Phys. 09 (2015) 023.

[61] N. Aghanim et al. (Planck Collaboration), Planck 2018 results. VI. Cosmological parameters, arXiv:1807.06209.

[62] F. Kahlhoefer, K. Schmidt-Hoberg, T. Schwetz, and S. Vogl, Implications of unitarity and gauge invariance for simplified dark matter models, J. High Energy Phys. 02 (2016) 016.

[63] Maxima, Maxima, a computer algebra system. version 5.41.0, 2017.

[64] E. L. Woollett, Dirac2: A high energy physics package for maxima, 2012.

[65] B. W. Lee, C. Quigg, and H. B. Thacker, Weak interactions at very high-energies: The role of the Higgs boson mass, Phys. Rev. D 16, 1519 (1977). 